\title{
A co-simulation approach to the wheel-rail contact with flexible railway track
}

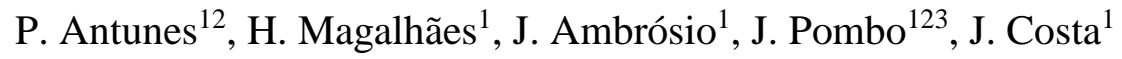 \\ ${ }^{1}$ IDMEC, Instituto Superior Técnico, Universidade de Lisboa, Lisboa, Portugal \\ \{pedro.antunes,hugomagalhaes,jorge.ambrosio,joao.n.costa\}@ tecnico.ulisboa.pt \\ ${ }^{2}$ University of Huddersfield, Huddersfield, UK. \\ \{p.antunes,j.pombo\}@hud.ac.uk \\ ${ }^{3}$ ISEL, IPL, Lisboa, Portugal.
}

Accepted for publication in Multibody System Dynamics (18 August 2018):

Link: $\quad$ https://link.springer.com/article/10.1007\%2Fs11044-018-09646-0

DOI: $\quad$ https://doi.org/10.1007/s11044-018-09646-0

Online ISSN: 1573-272X

Cite this article as: Antunes, P., Magalhães, H., Ambrósio, J. et al. Multibody Syst Dyn (2018). https://doi.org/10.1007/s11044-018-09646-0

\begin{abstract}
The standard approach to railway vehicle dynamic analysis includes running the vehicle multibody models in rigid railway tracks. The wheel-rail contact, independently of the rolling contact model used, is either handled online or via lookup tables. This traditional approach disregards the coupling effects between the railway vehicle dynamics and the railway track flexibility. In this work the assumption of rigidity of the railway track is released and a finite element model of the complete track, i.e., rails, pads, sleepers, ballast and infrastructure, is used to represent the track geometry and flexibility. A rail-wheel contact model that evaluates the contact conditions and forces is used online. The dynamics of the railway vehicle is described using a multibody methodology while the track structure is described using a finite element approach. Due to the fact that not only the multibody and the finite element dynamic analysis use different integration algorithms but also because the vehicle and track models are simulated in different codes a co-simulation procedure is proposed and demonstrated to address the coupled dynamics of the system. This approach allows to analyse the vehicle dynamics in a flexible track with a general geometry modelled with finite elements, i.e., including curvature, cant, vertical slopes and irregularities, which is another novel contribution. The methodology proposed in this work is demonstrated in an application in which the railway vehicle-track interaction shows the influence of the vehicle dynamics on the track dynamics and vice-versa.
\end{abstract}

Keywords Rolling contact, multibody vehicle model, flexible track, railway dynamics, online contact detection

\section{Introduction}

The development of computer resources favoured numerical dynamic analysis methods to become an essential part of the design and research process of railway systems. The quest for novel solutions to answer the increasing demands for network capacity, either by increasing the traffic speed or the axle loads, put pressure on the existing infrastructures that find in the computational analysis of potential solutions a tool for their virtual testing. The European 
Strategic Rail Research Agenda [1] and the European Commission for Transports white papers [2] have identified these topics as key scientific and technological priorities for rail transport over the next 20 years. One of the points emphasized is the need to reduce the cost of approval for new vehicles and infrastructure products with the introduction of virtual certification. Certainly, an important issue arising during the design phase of new railway vehicles is the improvement of their dynamic performance. The concurrent use of different computational tools allows carrying several simulations, under various scenarios, to reach optimized designs. Studies to evaluate the impact of design changes or failure modes risks can be performed in a much faster and less costly way than the physical implementation and test of those changes in real prototypes.

Current computer codes for railway applications use specific methodologies that, in general, either handle the vehicle dynamics on a rigid track or deal with moving loads on flexible track. By analysing such phenomena independently, it is not possible to capture all the dynamics of the complete railway system and relevant coupling effects. However, developing innovative and more relevant comprehensive methodologies, in a co-simulation environment, allow not only to integrate all physical phenomena, but also to assess the cross influence between them. Co-simulation procedures form a generalist approach of simulating coupled systems on a time depended basis [3-5]. As the dynamic analysis of multi-disciplinary models is often composed by sub-systems, co-simulation exploits this modular structure by addressing each sub-system with its own distinct formulation and time integration method. Co-simulation approaches avoid the use of a unique and complex formulation with a unified time integration method that compromises the accuracy of the dynamic analysis of each sub-system consequently becoming computationally expensive and time intensive. A wide range of applications use efficiently co-simulation to couple systems with different formulations, i.e., multidisciplinary problems [6-13]. There are also applications where co-simulation is employed to improve computational performance by allowing parallel computation $[14,15]$, or establishing active control on mechatronic systems $[16,17]$, or enabling the use of third party applications [18]. In the realm of railway numeric analysis tools co-simulation implementations are seldom found. One existing application case is the analysis of the pantograph-catenary interaction, in which a co-simulation procedure has been developed with a finite element catenary model interacting with a multibody pantograph model [19-21]. Also, in the framework of railway vehicle dynamics a co-simulation approach is used to set active control on vehicle models with tilting $[22,23]$.

The work presented here purposes a co-simulation procedure for the dynamic analysis of vehicle-track interaction where the main objective is to account for track flexibility in the dynamic behaviour analysis of railway vehicles, which in turn, is reflected on the rolling contact of the rail-wheel interaction. Railway dynamics is a subject where contributions from a wide range of fields are required. Different modelling approaches are used, depending on the objective of the study. The importance of the modelling aspects for the vehicle and track, in the context of their interaction, is related with the frequencies of interest associated to the particular phenomena under study in a State-of-Art review by Knothe and Grassie [24]. Although that work mostly focus on noise and it does not address the track geometry, it already presents some of the important modelling aspects required for flexible tracks to achieve meaningful analysis results. When addressing the vehicle-track interaction, from a perspective of evaluating the dynamic behaviour of a railway vehicle, the usual and most popular approach is to model the vehicle using a multibody system formulation model being the track considered a rigid structure [25-27]. This methodology provides acceptable results for dynamic analysis on a perspective of vehicle behaviour for ride safety and comfort [28] These models are adequate to evaluate low frequency dynamic responses such as lateral stability and curving 
behaviour, as most of the high frequency excitation is filtered by the vehicles suspension, up to a certain point. Gialleonardo et al. [29] show that the track flexibility has a significant effect on the evaluation of the vehicle critical speed and in the wheel/rail contact forces. Dynamic effects at mid to high frequency ranges require the introduction of track flexibility [30]. Even in the low frequency domain track flexibility must be considered when its effects on the railway dynamics are significant, such as when the track is considered to be flawed [31,32], or switches and crossings are considered [33]. The work by Martinez-Casas et al. [34] shows the importance of considering the flexibility of the railway track, and also of the wheelset, in the interaction between vehicle and track. Although in their work only a single wheelset and a perfect circular track are considered it can be accepted that the interaction phenomena identified can be expected to be present in more general scenarios. Furthermore, as the wheelrail contact forces evaluation depends on the geometry of the wheel and the rail, as much as in the relative position between them, track flexibility must be considered when analysing the development of these rolling contact forces along the track. In scenarios with tangent tracks models, in which modal superposition is used to reduce the size of the finite element track model, Dietz, Hippmann and Schupp [6] present the implementation of a coupled vehicle-track dynamics in a commercial multibody code. Due to the use of a modal representation of the flexible track this approach cannot handle to full dynamics of the system without considering an excessive number of modes for the track, which not only leads to computational inefficiency but also prevents the introduction of nonlinear elements, localized deformations and more general geometries. To this end, the work by Zhai, Wang and Cai [35] demonstrates the importance of considering the coupled vehicle-track dynamics with flexible tracks by developing a simulation scenario, validated experimentally, in which the spatial vehicle multibody model operates in a two tracks, one with large radius and another with a small radius. However, in all the works cited here the track geometry is either a tangent track or a curved track with constant radius, never considering a more general, and realistic geometry.

In this work, a multibody formulation is used to model the railway vehicle and a finite element formulation is presented to model the railway track. To establish the interaction between these models a novel co-simulation procedure, able to handle the dynamics between the systems, is proposed. This approach allows to analyse the vehicle dynamics in a flexible track with a general geometry modelled with finite elements, i.e., including curvature, cant, vertical slopes and irregularities, which is another novel contribution that can be used not only to address the running scenarios studied in this work but also to contribute to a number of challenging engineering problems associated to the train-track interaction occurring in tracks with small radius curves such as squeal noise and short pitch corrugation. A comparative study on the dynamics of a multibody vehicle with rigid and with a flexible railway track is presented to appraise the coupled dynamics of the systems and the modification of the rolling contact of the wheel with the track rail.

\section{Railway Vehicle Multibody Model}

The vehicle multibody model is characterized by a set of rigid and/or flexible bodies that are interconnected by force elements and joints. In turn, the representation of the mechanical elements that constrain the relative motion between structural elements allows the modelling of the relative mobility of the system components. The equations of motion that represent a multibody model of a railway vehicle, depicted in Figure 1, are written together with the second time derivative of constraint equations as [36]: 


$$
\left[\begin{array}{cc}
\mathbf{M} & \boldsymbol{\Phi}_{q}^{T} \\
\boldsymbol{\Phi}_{q} & \mathbf{0}
\end{array}\right]\left\{\begin{array}{c}
\ddot{\mathbf{q}} \\
\boldsymbol{\lambda}
\end{array}\right\}=\left\{\begin{array}{c}
\mathbf{g} \\
\boldsymbol{\gamma}
\end{array}\right\}
$$

where $\ddot{\mathbf{q}}$ is the vector with the accelerations of the rigid bodies and $\lambda$ is the Lagrange multiplier vector associated to the joint reaction forces. The remaining terms are described in further detail hereafter.
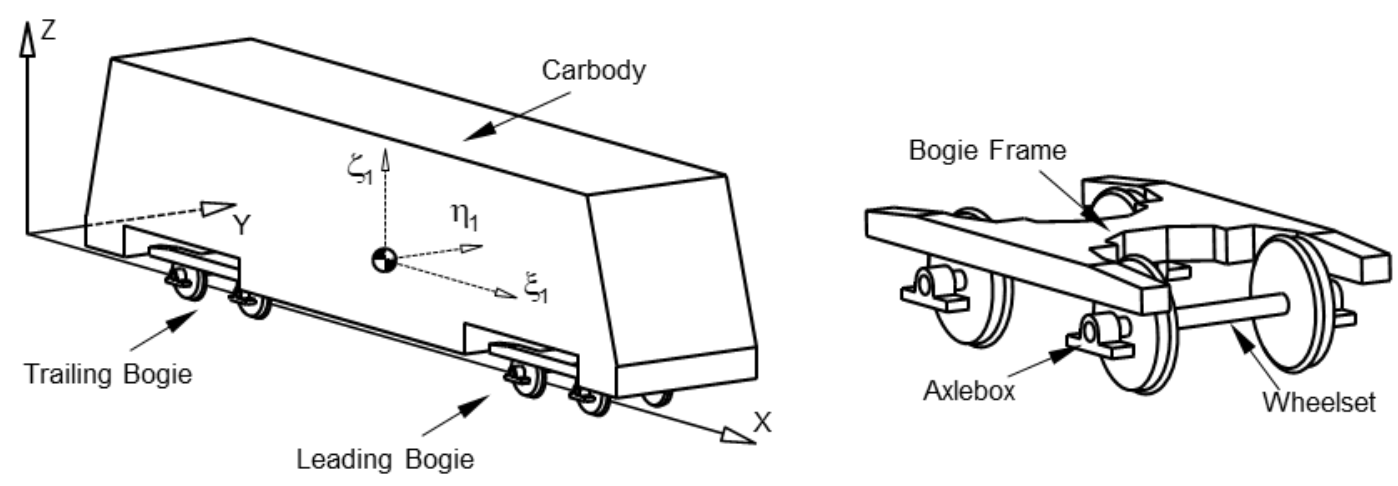

Figure 1: General multibody model of a railway vehicle.

The multibody model considered in this work comprises a carbody, bogie frames, wheelsets and axleboxes which are modelled as rigid bodies. Their mass and inertial properties are used to form the mass matrix M. The mechanical joints, in general, are modelled as kinematic constraints, being their modelling parameters associated to their geometric properties, which are used to form the constraint equations, whose second time derivative includes the Jacobian matrix, $\boldsymbol{\Phi}_{\mathbf{q}}$, and the right hand side vector, $\boldsymbol{\gamma}$. The primary and secondary suspension elements, depicted in Figure 2, are represented as springs and dampers with appropriate constitutive relations, being the forces transmitted to the connected bodies included in the force vector, $\mathbf{g}$. The wheel-rail contact forces are also included in the force vector, being their treatment described in Section 4 of this work.

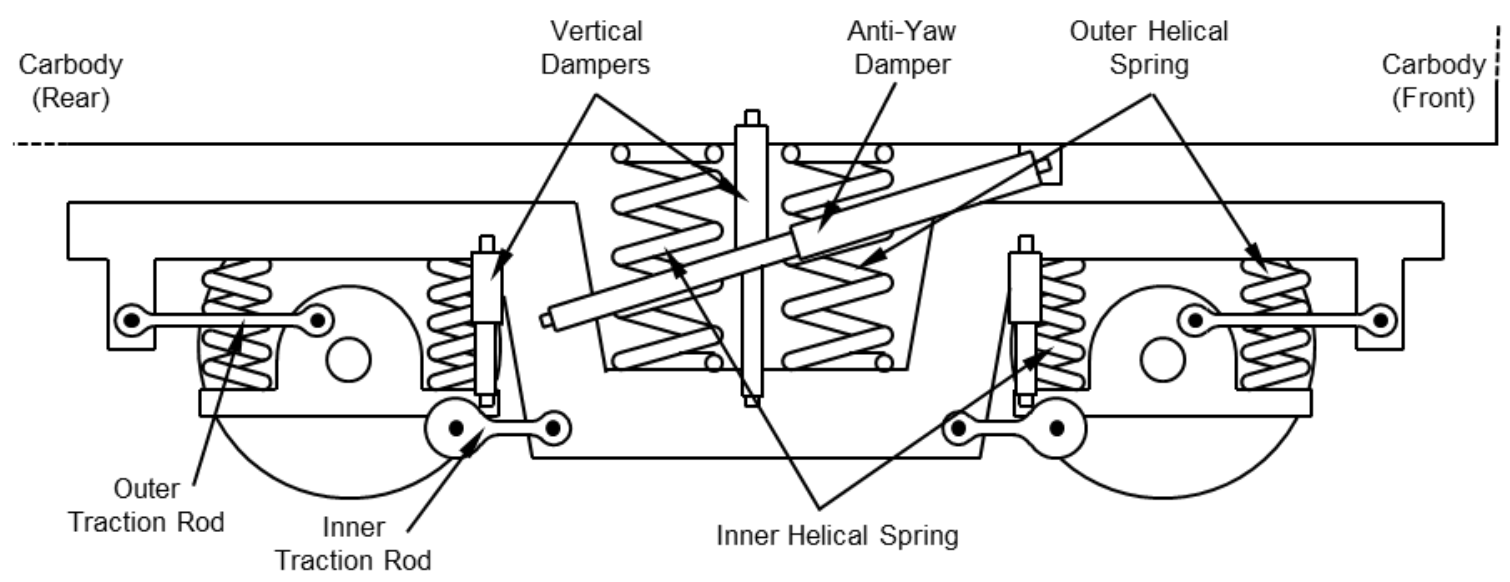

Figure 2: Suspension system of the railway vehicle.

The position and velocity constraint equations are not used explicitly in the integration of the system accelerations and velocities leading to a drift that results in the violation of these equations, as time progresses. It is necessary to eliminate or maintain the violations of the 
constraint equations under control. The kinematic constraint violations are stabilized using the Baumgarte stabilization method, while kept under prescribed thresholds, or eliminated by using a coordinate partition [37] when they exceed a pre-established value. The solution of the forward dynamics problem, for the multibody model, is obtained by using a variable time step and variable order numerical integrator [38].

\section{$3 \quad$ Track Finite Element Model and Equilibrium Equations}

The railway track is modelled using the finite element method being its dynamics analysed with suitable numerical methods. The ingredients of the finite element model are first described here being the systematic generation of the finite element model described afterwards. Finally, the equations of motion for the finite element model are presented.

\subsection{Finite element components}

The railway track is composed by several structural elements: rails, fasteners, rail pads, sleepers, ballast or slab and the substructure as depicted in Figure 3. In this work, the track model is assumed to have only linear deformations being its model built with linear finite elements. The rails and sleepers are modelled by three-dimensional beam elements, based on Euler-Bernoulli theory [39], the rail pads and fasteners and track supporting layers are modelled with 6 degrees of freedom spring-damper elements. A consistent mass matrix is used for the beam finite elements while a lumped mass description of the inertia is used for other elements in the model.
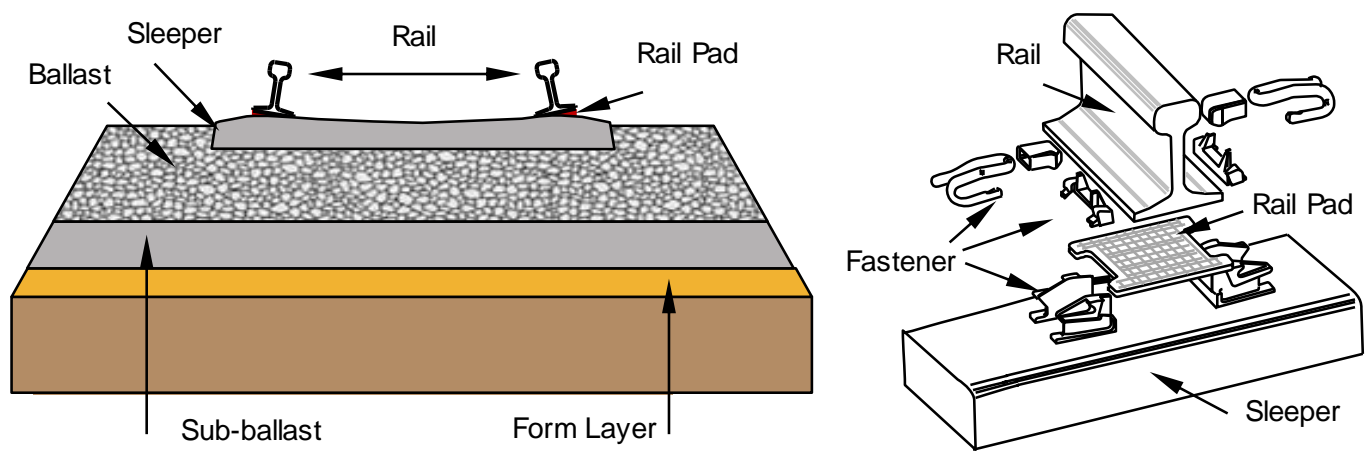

Figure 3: Typical construction of a railway track with its structural components: a) Track including the ballast and sub-structure b) Exploded view of the fixation of the rail to the sleeper

The rails are modelled with beam elements being 6 elements used between sleepers to ensure a proper geometry in curves. The sleepers are symmetric being the model of each one made of 5 beam elements to accommodate transitions of cross-section and/or material properties characteristic of these structural elements. The connection between the sleeper and the rail is modelled using a single spring-damper element with translational stiffness and damping along three perpendicular directions, which represents the sleeper pad, and rotational stiffness along the tangent direction of the rail, which is representative of the rail fastening system that prevents the rail from rotating. The track supporting layers are modelled considering two types of spring-damper elements: those connecting the sleepers to the foundation and those connecting two consecutive sleepers. The sleeper to foundation connection is represented by the vertical elements below the sleepers, depicted in Figure 4, and accounts for the flexibility of the supporting layers directly below the sleeper. The sleeper to 
sleeper connection represented by the in track-plane elements connecting the sleepers, as depicted in Figure 5, accounts for the interlocking action of the supporting structure, i.e., the ballast or the slab. The topology of the track model, with the structural elements considered, is well inline with the recommendations of Knothe and Grassie [24].

The track supporting layers consider translational stiffness and damping along three perpendicular directions. The foundation is modelled as a fixed "rigid" ground constraining the lower nodes of the track supporting layers finite element mesh. Finally, to avoid the elastic wave reflection characteristic of finite length models intended to represent infinite or very long tracks, massless spring-damper elements are added to the beginning and to the end of the railway track and constrained. This setup corresponds to energy absorption boundary conditions that dissipate the energy associated with the incoming elastic wave thus preventing its reflection, independently of the track length considered in each particular model of the track. The effectiveness of the absorption boundary conditions is achieved by selecting proper damping characteristics for the terminal spring-damper elements the elastic wave reflection is prevented.

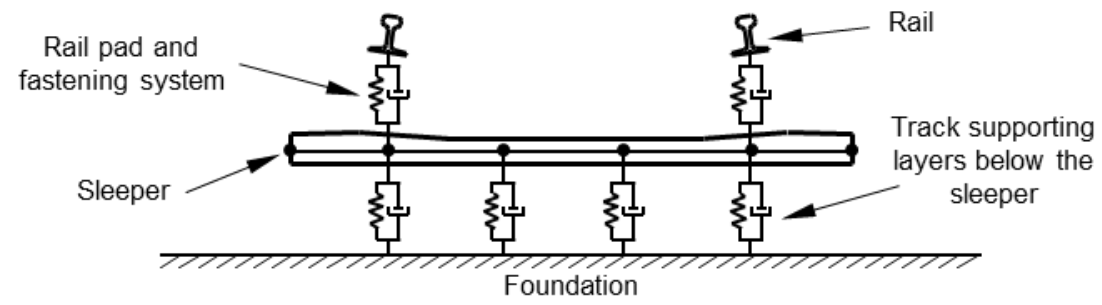

Figure 4: Cross section view of the track model.

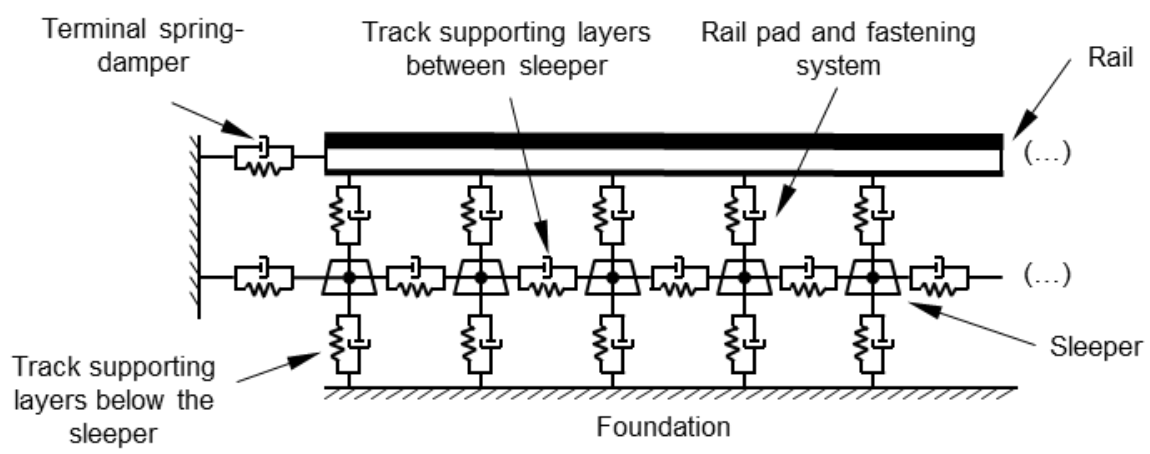

Figure 5: Longitudinal view of the track model.

\subsection{Systematic generation of the track finite element model}

The track geometrical description, based on the motion of a Frenet-Serret frame of the rails centreline curve is the basis of the finite element model construction used here [40, 41]. The information necessary to define the railway track centreline geometry, and the local plane in which the track must lay, is obtained from the curvature, cant and elevation information available for the description of the track geometry. The geometry and position of the rails is obtained from the track centreline geometry, taking into account the gauge and the rail geometry, using the track moving frame, as illustrated in Figure 6.

Using the geometric description of the left and right rails, as a function of their arc length, the position of the nodes of the rails, $\mathbf{r}_{L r}, \mathbf{r}_{R r}$, are defined as well as the local nodal coordinate frames $\left(\xi_{L r}, \eta_{L r}, \zeta_{L r}\right)$ and $\left(\xi_{R r}, \eta_{R r}, \zeta_{R r}\right)$, for the left and right rails respectively. The finite element mesh of the track includes nodes placed in planes for which the tangent vector to the track 
centreline is normal spaced such a way, along the centreline arc-length, that they include the sleepers, pads and fasteners, such as in the case illustrated in Figure 6 (a). In this case, there are two nodes associated with the rail cross-section center, six nodes along the sleepers to enable modelling monoblock, twin-block and timber sleepers, and four nodes for the track foundations. In-between sleepers, there are five rail nodes equally spaced along the rails curve. The beam finite element used for the rails have their cross-section oriented according to the local rail referential shown in Figure 6 (b). The remaining beam elements, used to model the sleepers depend on their geometry while the spring-damper elements used to represent the ballast resistance in the tangent-to-track plane and in its vertical direction are set in between the sleeper nodes and either the foundation or other sleeper nodes. For more details on the automatic track mesh construction the interested reader is directed to the work by Costa [42].

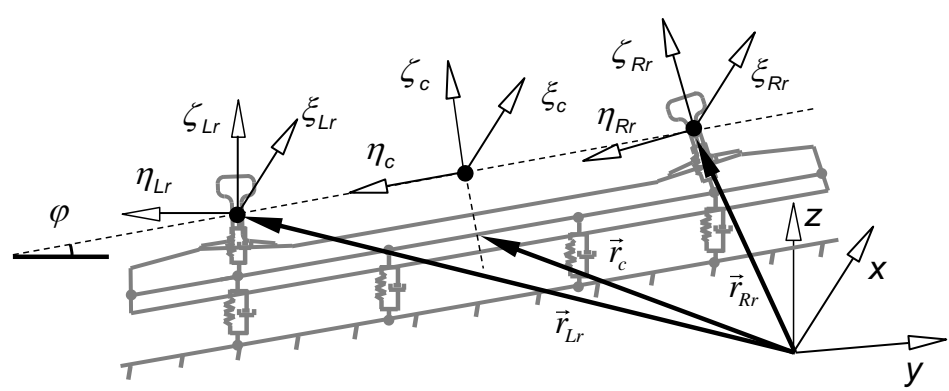

(a)

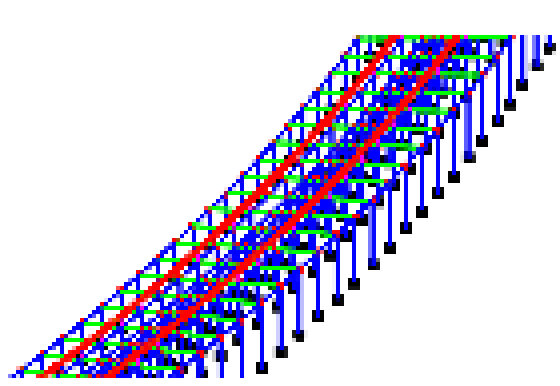

(b)

Figure 6: Elements of the finite element mesh of the track: (a) Position coordinates and local reference frame of the track and rails; (b) Finite element mesh for the railway track.

\subsection{Equations of motion of the track finite element model}

The dynamic equilibrium equations of a railway track are assembled and written as [43, 44]:

$$
\mathbf{M a}+\mathbf{C v}+\mathbf{K d}=\mathbf{f}_{\text {track }}
$$

where $\mathbf{M}, \mathbf{C}$ and $\mathbf{K}$ are the finite element global mass, damping and stiffness matrices, and $\mathbf{a}$, $\mathbf{v}, \mathbf{d}$ and $\mathbf{f}$ are the acceleration, velocity, displacement and force vectors, respectively. The global matrices $\mathbf{M}, \mathbf{C}$ and $\mathbf{K}$ are built by assembling the individual finite element matrices, according to the topology of the track mesh. The damping behaviour of the beam elements is represented using Rayleigh damping [44]. The force vector $\mathbf{f}_{\text {track }}$, containing the sum of all external applied loads, is evaluated at every time step of the integration as:

$$
\mathbf{f}_{\text {track }}=\mathbf{f}_{g}+\mathbf{f}_{c}
$$

where $\mathbf{f}_{g}$ represents the gravitational forces and $\mathbf{f}_{c}$ represents the equivalent wheel-rail contact forces and moments transferred from the application points to the finite element nodes, as described in detail in Section 4.3.

All matrices appearing in the left-hand side of Eq. (2) are constant, for the application scenarios foreseen in this work being, consequently, linear equations of motion. The dynamic behaviour of the track is solved using an integration algorithm based on the implicit Newmark trapezoidal rule [45]. This method is selected due to its unconditional stability, when used implicitly, and its proven robustness in FE applications, as the one performed in this work, [44]. 


\section{$4 \quad$ Wheel-Rail Contact}

In the vehicle-track co-simulation procedure, the coupling between both sub-systems is associated to the wheel-rail contact. The evaluation of the contact forces requires that the position and velocities of the flexible rail and rigid wheel are known and that a suitable contact force model is used. After evaluating the contact forces, these have to be transferred from their application points to particular points of the model components, i.e., the mass centers of the rigid bodies of the multibody model or the nodes of the finite element model.

\subsection{Wheel-rail contact model}

The rolling contact problem that characterizes the wheel-rail interaction is solved in two steps: the contact detection in which the contact points are identified, and; the contact force modelling in which the interaction forces involved are evaluated. The online wheel-rail contact detection method proposed by Pombo et al $[41,46]$ is the starting point for the approach proposed here.

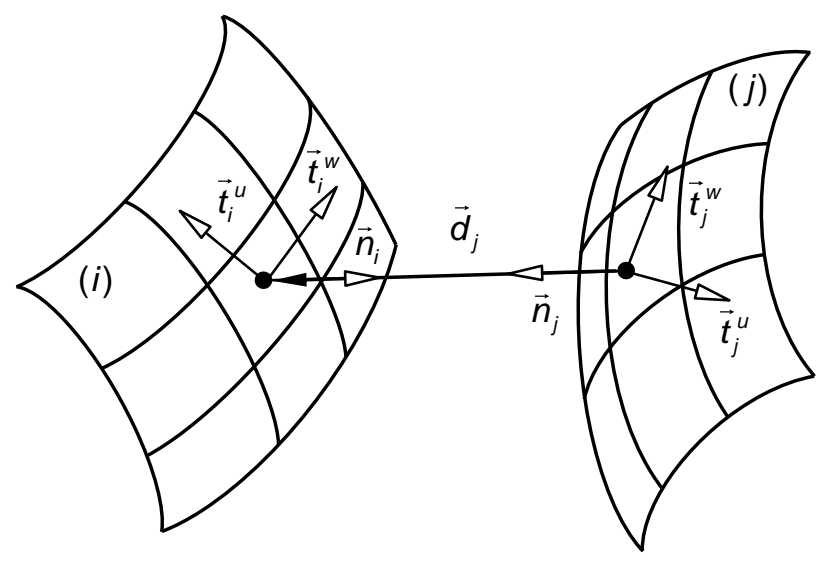

Figure 7: Contact detection between two surfaces [41, 46]

The wheel-rail contact detection problem is similar to the contact detection between two parametric surfaces, as those depicted in Figure 7, described by parameters $u_{i}, w_{i}, u_{j}$ and $w_{j}$. The location of the potential contact points in the surfaces must be such that the tangent planes to the surfaces, in those points, are parallel to each other. The surface parallelism condition is described by the nonlinear system of equations

$$
\left\{\begin{array}{l}
\mathbf{d}_{j}^{T} \mathbf{t}_{i}^{u}=0 \\
\mathbf{d}_{j}^{T} \mathbf{t}_{i}^{w}=0 \\
\mathbf{n}_{i}^{T} \mathbf{t}_{j}^{u}=0 \\
\mathbf{n}_{i}^{T} \mathbf{t}_{j}^{w}=0
\end{array}\right.
$$

where $\mathbf{d}_{j}$ is the distance vector between the potential points of contact, $\mathbf{n}_{i}$ and $\mathbf{n}_{j}$ are the normal vector of surfaces $i$ and $j, \mathbf{t}_{i}^{u}$ and $\mathbf{t}_{i}^{w}$ are tangential vectors of surface $i$ and $\mathbf{t}_{j}^{u}$ and $\mathbf{t}_{j}^{w}$ are tangential vectors of surface $j$, shown in Figure 7 , all defined as function of the surfaces parameters.

For each potential contact pair in the wheel-rail contact, i.e. the tread-rail and flange-rail contact pairs, contact exists if

$$
\mathbf{d}_{j}^{T} \mathbf{n}_{i}>0
$$


If contact exists in a particular contact pair, normal and tangential forces are calculated and applied to the bodies in contact on the contact points identified.

The interaction between the wheel and the rail is represented by the contact model proposed by Pombo et al [41, 46]. This model considers that the wheel surface is described by two parametric surfaces, for the tread and for the flange, while the rail is described by a single parametric surface. Therefore, two potential contact points may develop between wheel and rail, the tread-rail and the flange-rail contact points shown in Figure 8.
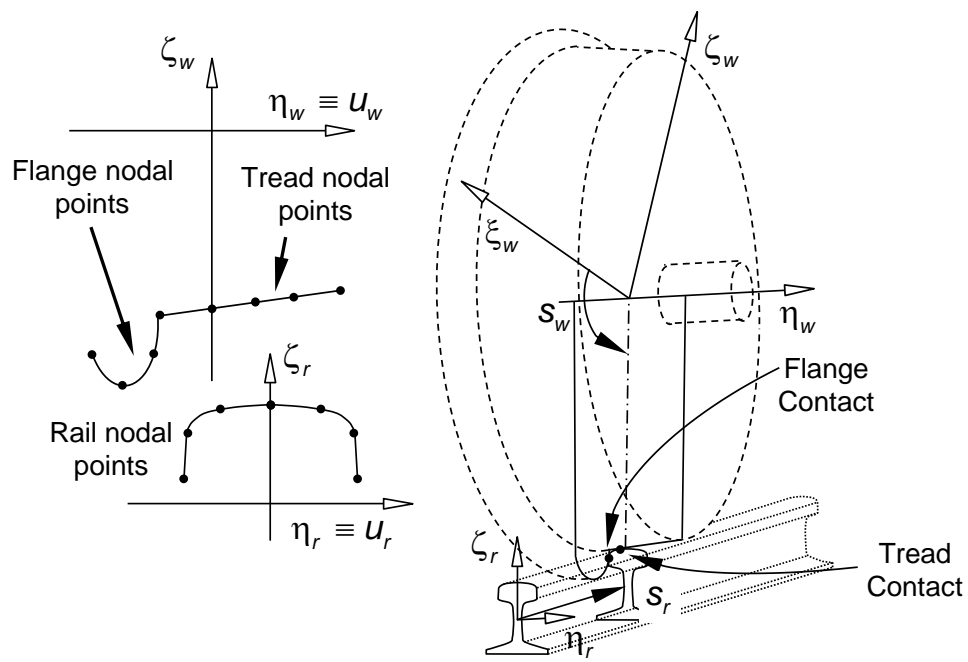

Figure 8: Identification of the parameters used in the wheel and rail parametric surfaces including the wheel tread and flange and rail profiles and surface parameters for the wheel $\left(s_{w}, u_{w}\right)$ and for the rail $\left(s_{r}, u_{r}\right)$.

The wheel profile is defined by two sets of nodal points, one for the tread and the other for the flange profile. These nodal points are interpolated to define the cross section of the wheel profile, as a function of parameter $u_{w}$, which in turn is rotated about the wheel axis $\eta_{w}$, with the angle $s_{w}$ starting from $\xi_{w}$, to form the parametric surface of revolution that defines the geometric shape of the wheel. The rail profile is also obtained by the interpolation of another set of nodal points, which are interpolated to define the rail cross-section, as a function of parameter $u_{r}$, which, in turn, is swept along the rail arc with the length of the sweep being defined by the arc-length $s_{r}$, starting from the origin of the rail. Consequently, the parametric surfaces of the wheel tread and flange and of the rail, depicted in Figure 8, are fully described by parameters $s_{w}, u_{w}, s_{r}$ and $u_{r}$ that play the role of parameters $u_{i}, w_{i}, u_{j}$ and $w_{j}$ in Eq. (4).

The effect of the flexibility of the track on the rail position and orientation is graphically shown in Figure 9(a), where a rail finite element is displaced with respect to its initial position, in grey, and for which the cross-sections are rotated relatively to their initial orientations. Let the finite element in which wheel-rail contact occurs connect node $i$ to node $j$, as shown in Figure 9(a). The position and orientation of the centre of the rail cross-sections in the beam finite element is related to the initial geometry, finite element nodal displacements and shape functions by

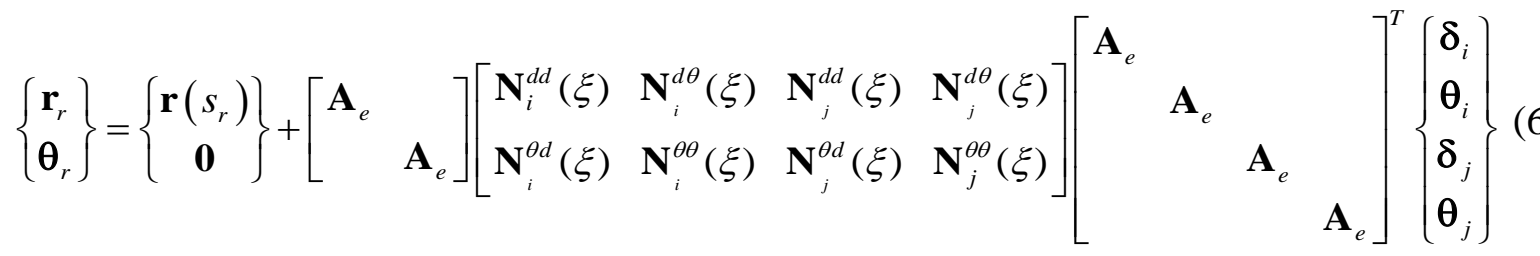

where $\mathbf{r}\left(s_{r}\right)$ is the position of the centre of the rail cross-section that includes the contact point 
for the rigid track, as described by Pombo et al $[41,46], \boldsymbol{\delta}_{i}$ and $\boldsymbol{\delta}_{j}$ the nodal displacements , $\boldsymbol{\theta}_{i}$ and $\boldsymbol{\theta}_{j}$ the nodal rotations, all expressed in the inertia frame coordinates, $\mathbf{A}_{e}$ is the finite element transformation matrix from local to global coordinates and $\mathbf{N}^{d d}, \mathbf{N}^{d \theta}, \mathbf{N}^{\theta d}$ and $\mathbf{N}^{\theta \theta}$ are submatrices with the shape functions of the beam element [39]. Eq. (6) is function of $\xi=\left(s_{r}-s_{i}\right) /\left(s_{j}-s_{i}\right)$, which is the parametric length coordinate of the finite element in which the contact takes place, being $s_{r}$ the arc-length of the rail up to the contact point and $s_{i}$ and $s_{j}$ the rail arc-lengths up to nodes $i$ and $j$, respectively.

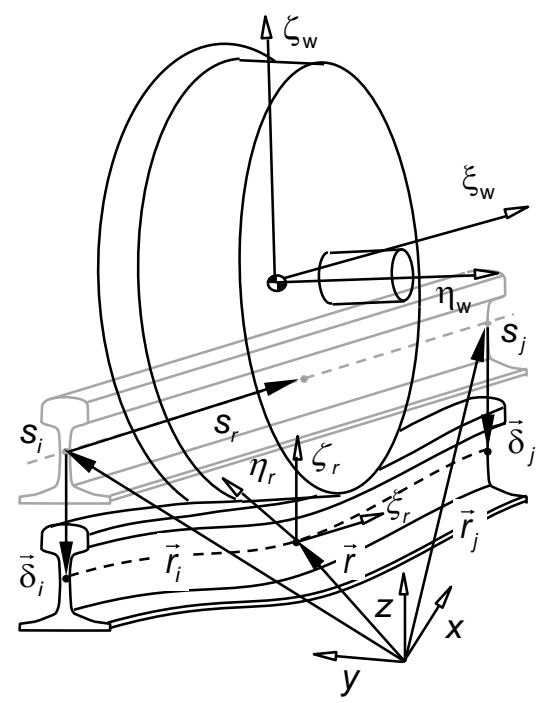

(a)

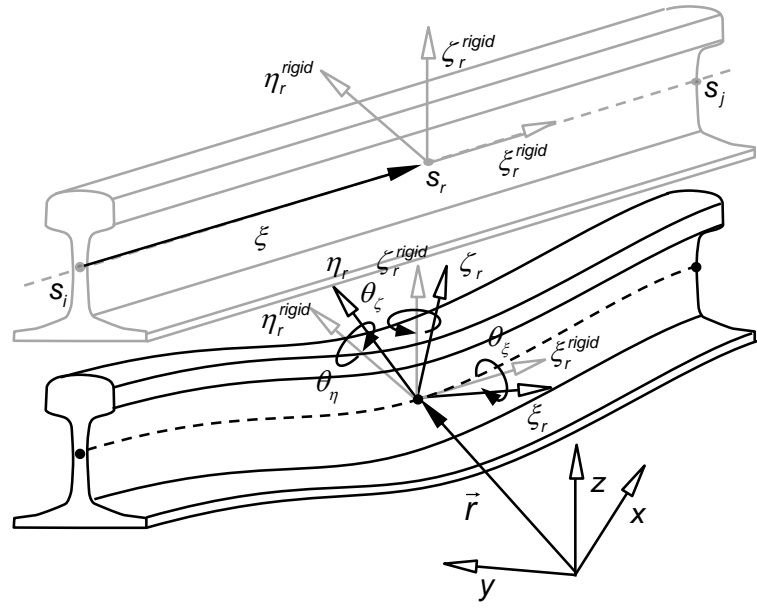

(b)

Figure 9: Deformation of the rail due to the wheel contact: (a) displacement of the rail cross-section that includes the contact point; (b) rotation of the rail cross-section.

Due to the rail deformation the rail cross-sections rotate with respect to their orientation on the rigid track, such a way that they remain perpendicular to the tangent of the arc line of their centres. The linear beam bending theory is used in the formulation of the linear beam finite elements being the infinitesimal rotations of a cross-section of the element, given, in Eq. (6), by $\boldsymbol{\theta}_{r}$. The transformation matrix from the rigid rail cross-section frame $(\xi, \eta, \zeta)_{r}{ }^{\text {rigid }}$ to the deformed rail cross-section frame $(\xi, \eta, \zeta)_{r}$, both shown in Figure $9(\mathrm{~b})$, is given by

$$
\mathbf{A}_{\theta}=\left[\begin{array}{ccc}
1 & -\theta_{\zeta} & \theta_{\eta} \\
\theta_{\zeta} & 1 & -\theta_{\xi} \\
-\theta_{\eta} & \theta_{\xi} & 1
\end{array}\right]
$$

The consequence of the displacement and rotation of the rail cross-section on the wheel tread and flange to rail contact searches is that not only the evaluation of vector $\mathbf{d}_{j}$ in Eq.(4) must take into account the new location of the centre of the cross-section $\mathbf{r}_{r}$ as given by Eq.(6) but also the rail surface vectors $\mathbf{n}_{j}, \mathbf{t}_{j}^{u}$ and $\mathbf{t}^{w}{ }_{j}$ need to be rotated. In the wheel-rail contact formulation with a rigid track, by Pombo et al $[41,46]$, the normal, bi-normal and tangent vectors of the left and right rails are pre-calculated and included in a table accessed online during the contact search. In the procedure for the flexible track the original vectors in the rigid track table are rotated by matrix $\mathbf{A} \theta$ and $\mathbf{r}_{r}$ is added to the rigid rail position before being used in the contact search algorithm, which is done by solving the system of nonlinear equations 


$$
\left\{\begin{array}{l}
\mathbf{d}_{j}^{T} \mathbf{t}_{i}^{u}=0 \\
\mathbf{d}_{j}^{T} \mathbf{t}_{i}^{w}=0 \\
\mathbf{n}_{i}^{T} \mathbf{A}_{\theta} \mathbf{t}_{j}^{u}=0 \\
\mathbf{n}_{i}^{T} \mathbf{A}_{\theta} \mathbf{t}_{j}^{w}=0
\end{array}\right.
$$

If Eq.(5) is fulfilled for a particular contact pair, normal and tangential contact forces need to be evaluated. These forces depend on the contact geometry and on the material properties of the wheel and rail. Assuming that the contact between the wheel tread or flange and the rail is non-conformal, the normal contact forces are calculated using an Hertzian contact force model with hysteresis damping is given by [47]

$$
f_{n}=K\left(1+\frac{3\left(1-e^{2}\right)}{4} \frac{\dot{\delta}}{\dot{\delta}^{(-)}}\right) \delta^{n}
$$

where $K$ is the stiffness coefficient, $e$ is the restitution coefficient, $n$ is a constant equal to 1.5 for metals, $\delta$ is the amount of interpenetration between the surfaces, $\dot{\delta}$ is the interpenetration velocity and $\dot{\delta}^{(-)}$is the relative velocity as impact starts.

The tangential forces are evaluated using the formulation proposed by Polach in which the longitudinal creep, or tangential, force is [48]

$$
f_{\xi}=f \frac{v_{\xi}}{v_{C}}
$$

while the lateral creep force is written as

$$
f_{\eta}=f \frac{v_{\eta}}{v_{C}}+f_{\eta S} \frac{\phi}{v_{C}}
$$

being $f$ the tangential contact force caused by longitudinal and lateral relative velocities between the contacting surfaces, generally designated as creepages in rolling contact, $v_{\xi}, v_{\eta}$ and $\phi$ are the longitudinal, lateral and spin creepages, respectively, in the point of contact, $v_{C}$ is the modified translational creepage, which accounts the effect of spin creepage and $f_{\eta S}$ is the lateral tangential force, or creep, caused by spin creepage. The Polach algorithm requires as input the normal contact force, the semi-axes of the contact ellipse, the combined modulus of rigidity of wheel and rail materials, the friction coefficient and the Kalker creepage and spin coefficients $c_{i j}$ [49].

The contact forces on the wheel tread and flange, shown in Figure 10 as vectors $\mathbf{f}_{t r, w}$ and $\mathbf{f}_{f l, w}$, respectively, are generically written as

$$
\mathbf{f}_{k, w}=f_{k, n} \mathbf{n}_{k}+f_{k, \xi} \mathbf{t}_{k, w}+f_{k, \eta} \mathbf{t}_{k, u} \quad k=t r, f l
$$

where $\mathbf{n}_{k}$ is the vector normal to the wheel surface, $\mathbf{t}_{k, w}$ is the tangent vector to the surface in the longitudinal direction of the wheel motion and $\mathbf{t}_{k, u}$ is the tangent vector in the lateral direction. In turn, the forces $\mathbf{f}_{t r, r}$ and $\mathbf{f}_{f l, r}$ represent the forces applied on the rails, which are opposite to those calculated for the wheels, i.e., $\mathbf{f}_{t r, w}$ and $\mathbf{f}_{f l, w}$.

\subsection{Wheel-rail contact model on vehicle}

In the multibody model, the information related to the wheel-rail contact forces is added to the force vector $\mathbf{g}$ in Eq. (1), in which all forces are supposed to be applied in the rigid bodies mass 
centres, i.e., the origin of the body fixed coordinate systems. The forces due to the wheel-rail contact are applied in the contact points of the wheelset, shown Figure 10 for the tread and flange contacts. Therefore, the contact forces are first transferred to the centre of the wheelset by adding all the contact forces to a force resultant and a transport moment due to the transference of the points of application to the wheel centre, as

$$
\begin{aligned}
& \mathbf{f}_{w h e e l}=\mathbf{f}_{t r, w}+\mathbf{f}_{f l, w} \\
& \mathbf{n}_{w h e e l}^{\prime}=\tilde{\mathbf{s}}_{t r, w}^{\prime} \mathbf{A}_{w s}^{T} \mathbf{f}_{t r, w}+\tilde{\mathbf{s}}_{f l, w}^{\prime} \mathbf{A}_{w s}^{T} \mathbf{f}_{f l, w}
\end{aligned}
$$

where $\mathbf{s}_{t r, w}^{\prime}$ and $\mathbf{s}_{f l, w}^{\prime}$ are the position vectors of the tread and flange contact points with respect to the wheel center and expressed in the wheelset body coordinate frame, and $\mathbf{A}_{w s}$ is the transformation matrix from the wheelset body frame to the inertia frame.
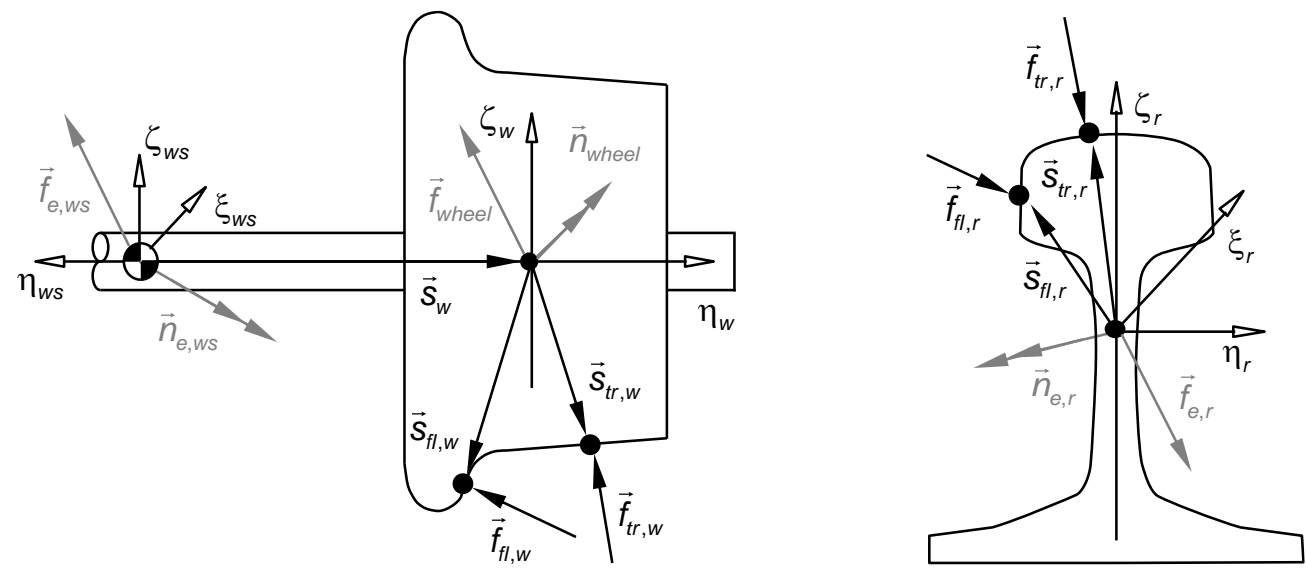

Figure 10: Wheel and rail contact forces, points of contact and equivalent forces and moments in the wheel center and in the rail cross-section center.

In the most common applications the wheels on the same wheelset are not independent, and consequently they are part of a single rigid body designated by wheelset. Therefore, the resultant force applied in the wheel mass centre is transferred to the wheelset mass centre, being the resultant force and transport moment on the wheelset due to the wheel-rail contact given by

$$
\begin{aligned}
& \mathbf{f}_{e, w s}=\mathbf{f}_{\text {wheel }} \\
& \mathbf{n}_{e, w s}^{\prime}=\tilde{\mathbf{s}}_{w}^{\prime} \mathbf{A}_{\text {ws }}^{T} \mathbf{f}_{\text {wheel }}+\mathbf{n}_{\text {wheel }}^{\prime}
\end{aligned}
$$

where $\mathbf{s}_{w}^{\prime}$ is the position of the wheel centre with respect to the wheelset mass centre, expressed in the wheelset body fixed coordinate system. Thus, the contribution of the wheel-rail contact forces to the force vector $\mathbf{g}$ of Eq. (1) is simply $\mathbf{g}_{e, w s}=\left[\mathbf{f}^{T}{ }_{e, w s}, \mathbf{n}^{\prime T}{ }_{e, w s}\right]^{T}$.

\subsection{Wheel-rail contact model on track}

In a finite element model lumped forces, such as the wheel-rail contact forces, can be applied on the nodes of the mesh but not in the middle of the element. As seen in Figure 10, the wheelrail contact forces applied on the rail surface whereas the beam element used in the model for the rail considers only its geometric centre. Therefore, the resultant of the contact forces, $\mathbf{f}_{e, r}$, is applied on the rail cross-section centre and a transport moment, $\mathbf{n}_{e, r}$, shown in Figure 10 and in Figure 11(a), is added to obtain the equivalent force system in the cross-section center as 


$$
\begin{aligned}
& \mathbf{f}_{e, r}=\mathbf{f}_{t r, r}+\mathbf{f}_{f l, r} \\
& \mathbf{n}_{e, r}=\tilde{\mathbf{s}}_{t r, r} \mathbf{f}_{t r, w}+\tilde{\mathbf{s}}_{f l, r} \mathbf{f}_{f l, w}
\end{aligned}
$$

where $\mathbf{s}_{t r, r}$ and $\mathbf{s}_{f l, r}$ are the contact position vectors with respect to the cross-section center, defined the inertia reference frame coordinates. Note that the transformation of coordinates of the contact position points from rail cross-section coordinates to global coordinates is done by $\mathbf{s}_{t r, r}=\mathbf{A}_{r} \mathbf{s}_{t r, r}^{\prime}$ and $\mathbf{s}_{f l, r}=\mathbf{A}_{r} \mathbf{s}_{f l, r}^{\prime}$ with the transformation matrix $\mathbf{A}_{r}=\left[\begin{array}{lll}\mathbf{u}_{\xi} & \mathbf{u}_{\eta} & \mathbf{u}_{\zeta}\end{array}\right]_{r}$.

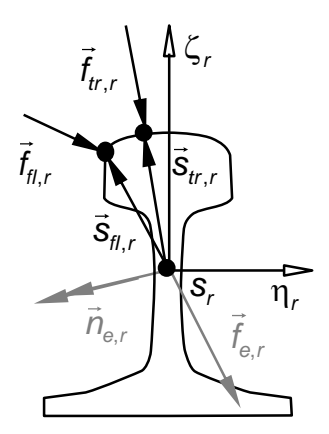

a)

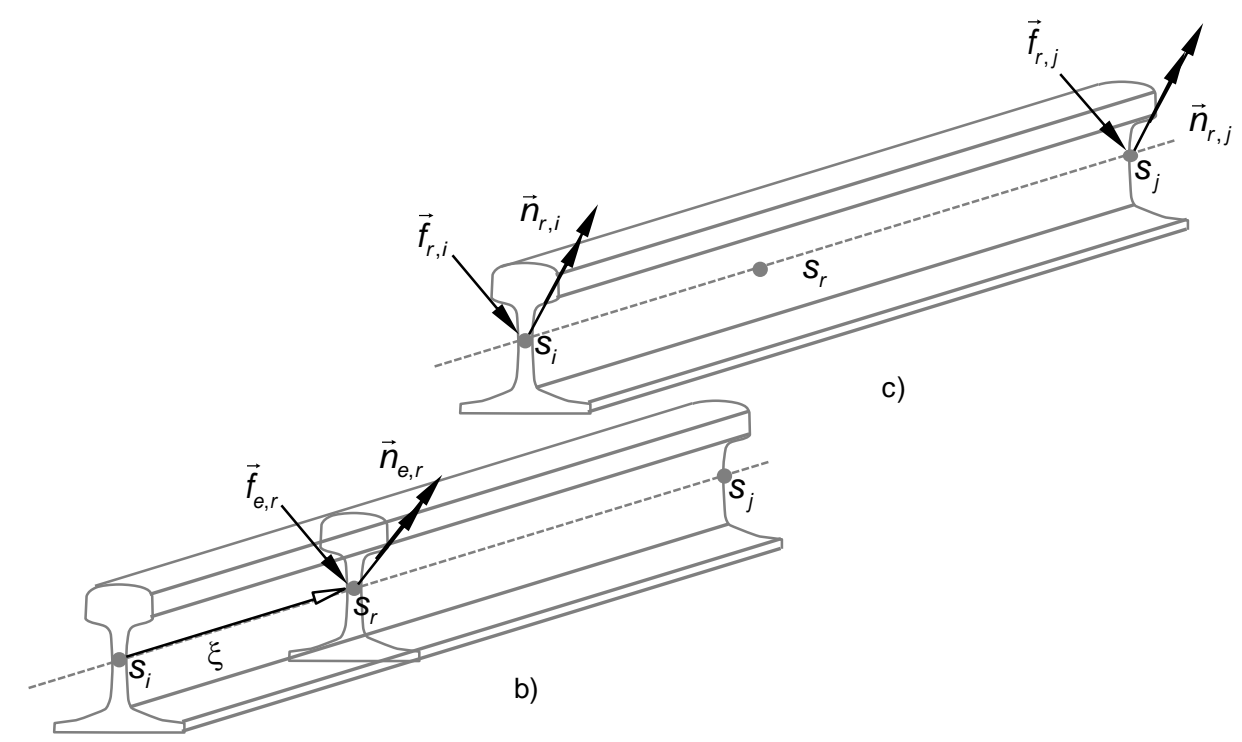

Figure 11: Wheel-rail contact force: (a) Rail cross-section in which the wheel tread and flange contact forces are applied; (b) Equivalent force system in the center of the cross-section; (c) Equivalent system of nodal forces in a particular finite element of the rail.

An equivalent system of forces and moments applied in the beam finite element nodes, shown in Figure 11, that represents contact forces and transport moment applied to the railcross-section centre needs to be evaluated. The equivalent nodal forces are related to the concentrated forces and moments via the shape functions matrix as

$$
\left\{\begin{array}{l}
\mathbf{f}_{r, i} \\
\mathbf{n}_{r, i} \\
\mathbf{f}_{r, j} \\
\mathbf{n}_{r, j}
\end{array}\right\}=\left[\begin{array}{llll}
\mathbf{A}_{e} & & & \\
& \mathbf{A}_{e} & & \\
& & \mathbf{A}_{e} & \\
& & & \mathbf{A}_{e}
\end{array}\right]\left[\begin{array}{llll}
\mathbf{N}_{i}^{d d}(\xi) & \mathbf{N}_{i}^{d \theta}(\xi) & \mathbf{N}_{j}^{d d}(\xi) & \mathbf{N}_{j}^{d \theta}(\xi) \\
\mathbf{N}_{i}^{\theta d}(\xi) & \mathbf{N}_{i}^{\theta \theta}(\xi) & \mathbf{N}_{j}^{\theta d}(\xi) & \mathbf{N}_{j}^{\theta \theta}(\xi)
\end{array}\right]^{T}\left[\begin{array}{ll}
\mathbf{A}_{e} & \\
& \mathbf{A}_{e}
\end{array}\right]^{T}\left\{\begin{array}{l}
\mathbf{f}_{e, r} \\
\mathbf{n}_{e, r}
\end{array}\right\}
$$

and applied on the finite element nodes, i.e., $\mathbf{f}_{r, i}$ and $\mathbf{n}_{r, i}$ are applied on node $i$ while $\mathbf{f}_{r, j}$ and $\mathbf{n}_{r, j}$ are applied on node $j$, as shown in Figure 11(c). The forces and moments are expressed in the inertia coordinate frame coordinates.

\section{$5 \quad$ Vehicle-Track Co-Simulation}

The vehicle-track co-simulation procedure, presented here, establishes the interaction between the individual sub-systems, each with its own distinct mathematical formulation and integration methodology, being their dynamic analysis performed by independent codes able to, eventually, run in a stand-alone mode. The behaviour of the two sub-systems is affected reciprocally by each other. A particular aspect of the co-simulation procedure proposed 
concerns the synchronisation of the integration algorithms that run with independent time steps, being the numerical stability and accuracy of the dynamic analysis of the coupled systems a fundamental aspect to account for [50].

The co-simulation procedure proposed is structured on three main key steps, addressed hereafter. The first step is to establish the coupling approach, i.e., an interface between the subsystems that defines a set of state variables or forces within each sub-system to be shared with the other. The second step is to establish a fast and reliable data exchange procedure for the state variables and forces. The third, and final, step is to build a communication protocol that manages the use of the state variables and contact forces through the integration scheme for both sub-systems during their dynamics analysis.

\subsection{Vehicle-track interface}

Though the coupling approach depends on the type of interaction between the models, most often the coupling is set by imposing either a kinematic constraint between the models or a set of constitutive interaction laws. Such constitutive interaction laws can result on a set of forces/torques that are applied on each sub-system. In this work, due to the nature of the coupled problem where their interaction is defined by the wheel-rail contact, the coupling of the sub-systems is established by the application of the resulting contact forces/torques on each model. Thus, each computer code solves its own equations of motion, which include the interaction forces. As the wheel-rail contact forces provide the link between to two subsystems, the evaluation of the contact is done in one of the sub-systems while the other provides the parameters required to make such evaluation possible, in this case the state variables that allow for the solution of the contact problem. Evaluating the wheel-rail contact on the track sub-system, as shown in Figure 12, avoids a computationally expensive communication scheme. The contact model requires the deformed centre position of the rails, in the neighbourhood of the arc length of the track in which contact occurs, $s_{r}$, to allow for the solution of the nonlinear Eq. (4) for contact detection, which in turn requires all information associated to the finite element mesh of the rails already available in the track sub-system. The vehicle sub-system is set to provide the spatial position, $\mathbf{q}_{w}$, and velocity, $\dot{\mathbf{q}}_{w}$, of each wheel centre of the vehicle model. The wheel-rail contact problem is solved in the track sub-system and, in return, the vehicle sub-system receives from the track sub-system an equivalent wheel-rail contact force, $\mathbf{f}_{e, w}$, and transport moment, $\mathbf{n}_{e, w}^{\prime}$, to be applied at the corresponding wheel centres. 

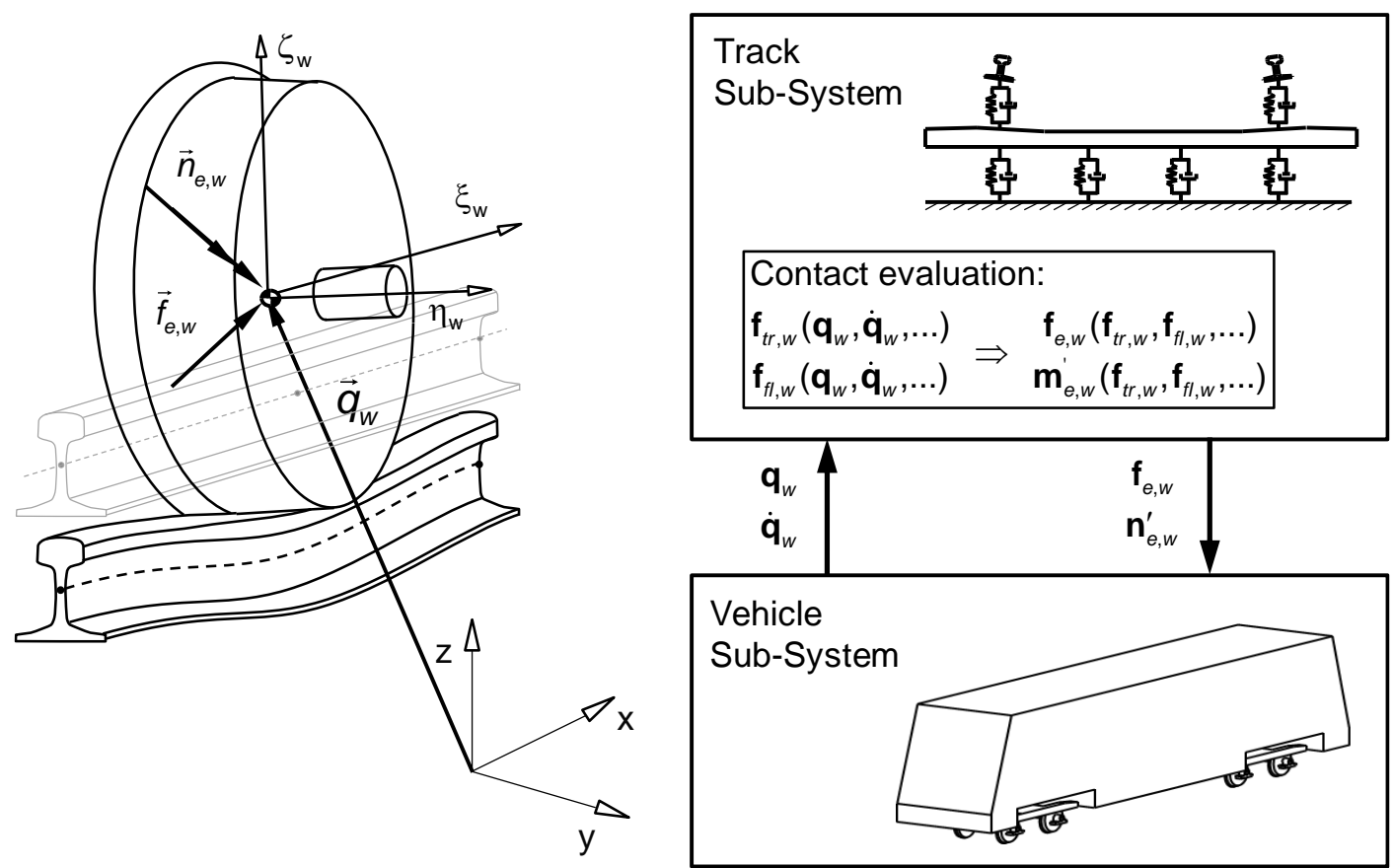

Figure 12: Vehicle-track co-simulation interface.

\subsection{Data exchange method}

As the state variables are a common resource shared between two concurrent processes being the data exchange procedure critical in the co-simulation. This procedure is not only responsible for exchanging the state variable data between sub-systems but also to control their access. This leads to two important requirements that the data exchange method needs to fulfil. First, given the frequency at which data needs to be exchanged, it must be sufficiently fast so that it does not become a bottleneck of the co-simulation procedure. Second, it must be robust by providing a mechanism where both sub-systems are synchronized over time and do not overstep each other.

The data exchange method is built by exchanging two communication files, as depicted in Figure 13. One file includes the state variables data, composed of the wheel centre position and velocity, denoted by V2T file, written by the vehicle sub-system code and read by the track sub-system code. The other file written by the track sub-system code and read by the vehicle sub-system code, denoted by $T 2 V$ file, includes the equivalent wheel-rail contact forces to be applied on the centre of each wheel. In order to keep both sub-systems synchronised and to avoid data do be overwritten without being read first, which is known as a race condition [51, 52], a binary semaphore is implemented [53]. Here, each communication file also carries a binary flag that according to its value either gives permission to one sub-system to read the data or the other to write it over. This method not only controls the reading/writing access of the state variables but also provides means to control the progress of the integration algorithms of each one of the individual analysis codes so that they stay synchronized. 


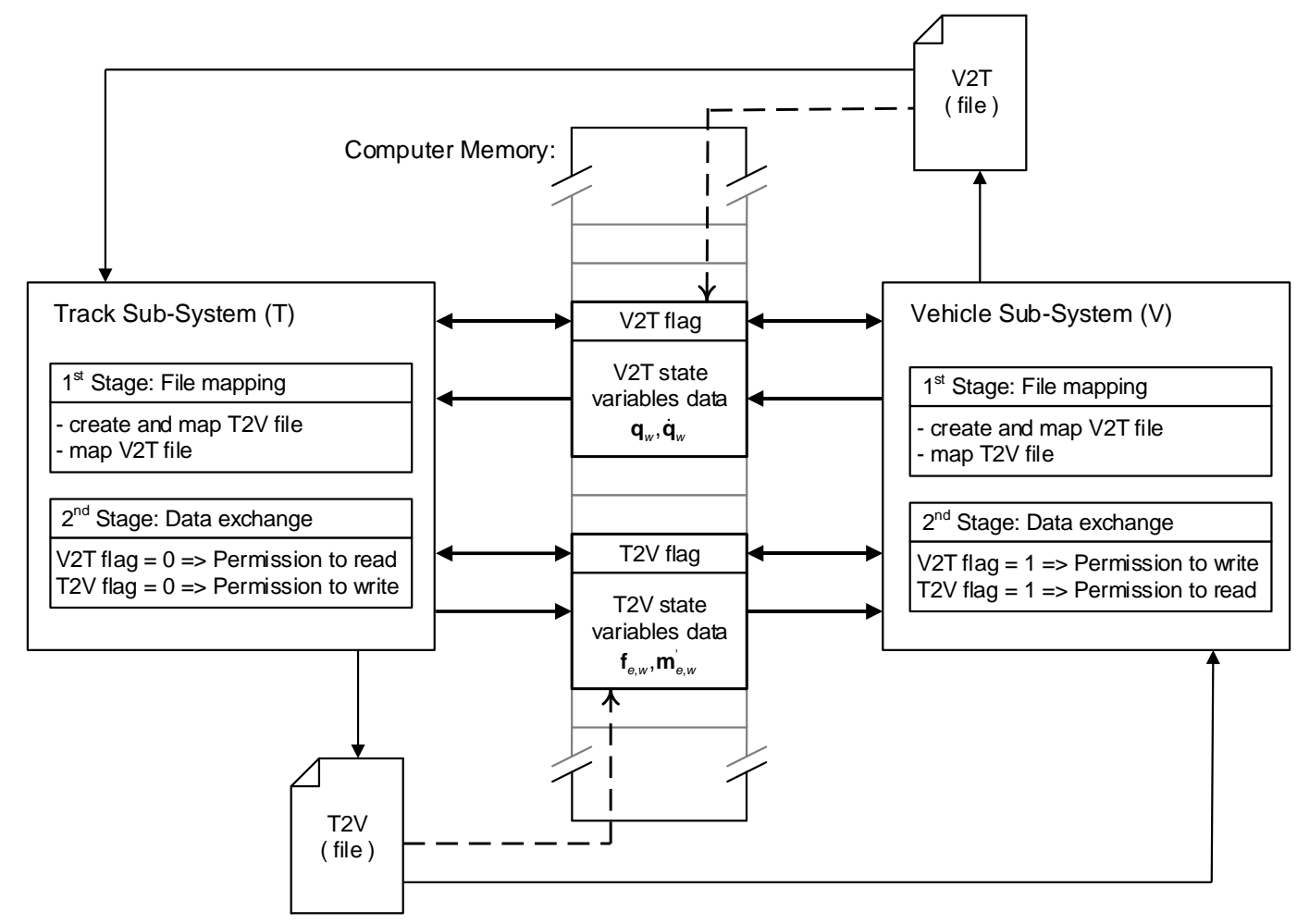

Figure 13: Vehicle-track data exchange procedure.

The time spent on data exchange between codes must be negligible compared to the computation time costs of the independent analyses. Therefore, the data exchange procedure uses memory sharing via memory mapped files. A memory mapped file is a segment of computer memory which is mapped in order to have a direct byte-for-byte assignment to a hard disk file or other resource that the operating system can refer to. Once this correlation is established, or mapped, the memory mapped file can be accessed directly from computer memory becoming a much faster data exchange process. This memory sharing implementation is depicted in Figure 13. At the start of the analysis, one of the applications creates a file and maps it to memory while the other waits for the file to be created. Whenever this file is found by the waiting application the file is also mapped to the same corresponding memory address. Having both applications mapped the same file in memory they can communicate using a common memory address whereas the created file only serves as a point of reference for both applications to map the same dataset in memory.

\subsection{Communication protocol}

The communication protocol is responsible for managing the use and update of the state variables along the integration scheme of each sub-system. In this work each sub-system has a distinct formulation and integration procedure, on one side the railway multibody vehicle model is evaluated as a nonlinear dynamic system handled with a variable time step, multiorder integrator, while a finite element track model is evaluated as dynamic linear system integrated with a Newmark family numerical integrator with a fixed time step. The heterogeneity of these integration schemes and the premise to keep them independent and fundamentally unchanged requires careful consideration. Thus, the compatibility between the two integration algorithms imposes that the state variables of the two sub-systems are readily 
available at every time step. This is guaranteed by a state variable interpolation/extrapolation scheme where the state variable data used by each sub-system is updated following the communication protocol presented in Figure 14. At a given time step, $t_{T}$, the track model requires the positions and velocities of the wheel centres to evaluate the wheel-rail contact force. Meanwhile, the vehicle model, evaluated with a variable time step, requires the equivalent wheel-rail contact forces available to be applied on its model and proceed with its integration. Therefore, there is the need of one of the sub-systems to make a prediction on a forthcoming time, before advancing to a new time step. Given the integration procedure structure between the two systems, the vehicle model is selected to be the leading sub-system. Hence, the equivalent contact forces to be applied on the wheel are estimated by linear extrapolation of the state variable data, $\mathbf{f}_{E}, t_{E}$, and provided by the track sub-system. Whenever the track sub-system integrator requires data to proceed it is set to wait until the vehicle model has advanced to the point where it can interpolate the results of its evaluation in order to provide the wheel positions and velocities for the required time step. It is important to note that the accuracy and stability of this methodology relies on ensuring that the vehicle sub-system variable time step size is never larger than the fixed time step of the track. Furthermore, the vehicle integrator time step size is also required to be small enough so it does not critically overextend the state variable extrapolation. This is guaranteed by limiting its maximum step size to be smaller than the track time step size.

\section{Demonstrative Application}

The demonstration of the vehicle-track co-simulation procedure proposed here, and of its implications on the wheel-rail rolling contact problem, is carried with a case scenario. Three alternatives are tested for the representation of the wheel-rail interaction problem. One corresponds to the co-simulation procedure, presented here, where a multibody vehicle model is coupled with a finite element track model so that track flexibility is taken into consideration. A second alternative consists of the same co-simulation procedure but assuming the track to be rigid by neglecting the finite-element nodal displacements. The third simulation is run, to serve as a control, with the standalone multibody code where the vehicle runs on the rigid track, i.e., using the standard approach in railway vehicle dynamics studies. 


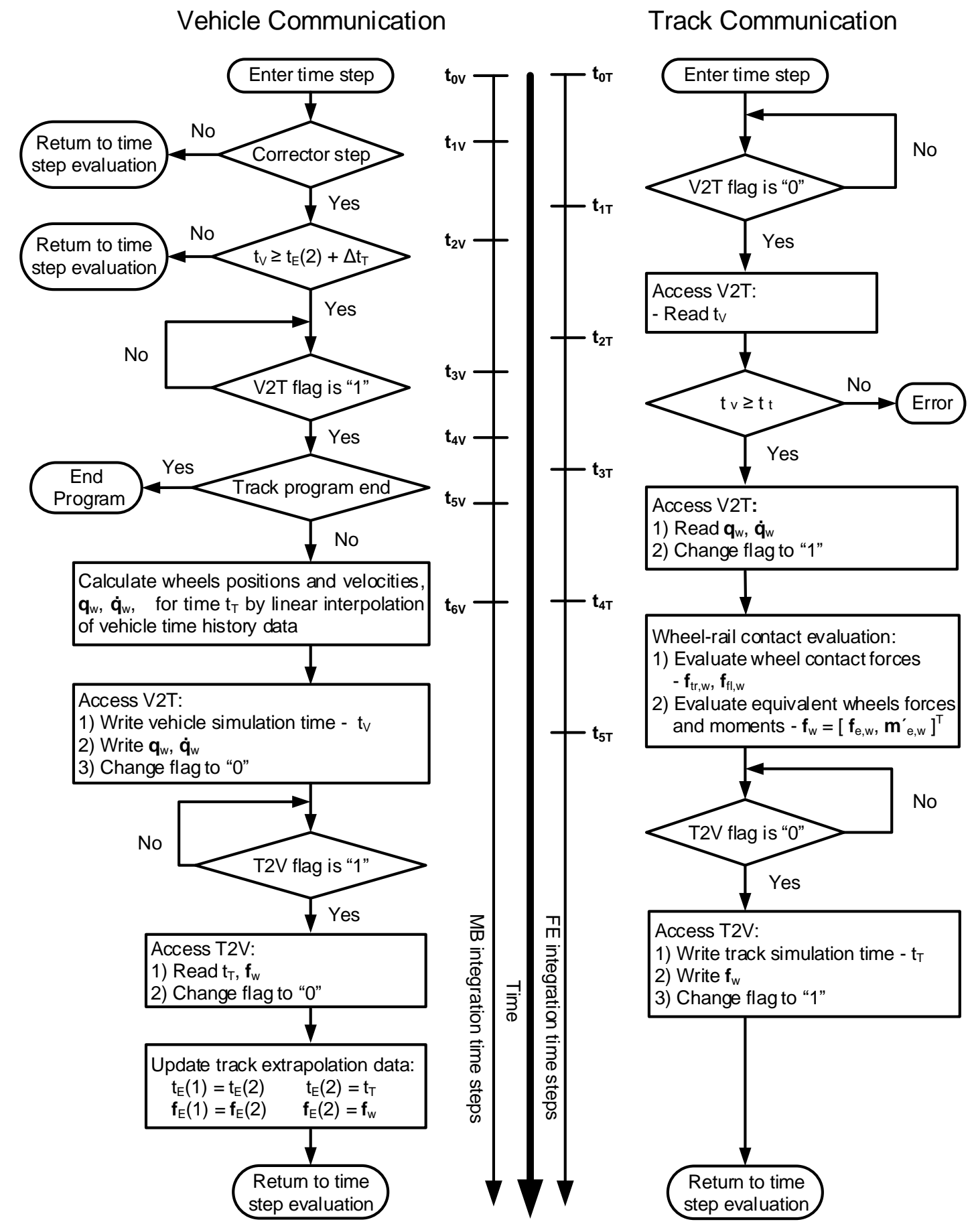

Figure 14: Vehicle-track communication protocol.

\subsection{Case scenario}

The track considered for the case scenario is composed by a straight segment followed by a small radius left-hand curve and a short straight track segment. It also includes two transition zones between the curve and straight segments as depicted in Figure 15. The track geometry is designed following standard EN13803-1 for a vehicle operating at a speed of $110 \mathrm{~km} / \mathrm{s}$ while 
negotiating a $500 \mathrm{~m}$ radius curve at its maximum allowed superelevation and cant deficiency limit. Iberic gauge is selected for the track with UIC60 rail profiles and 1/20 rail inclination.

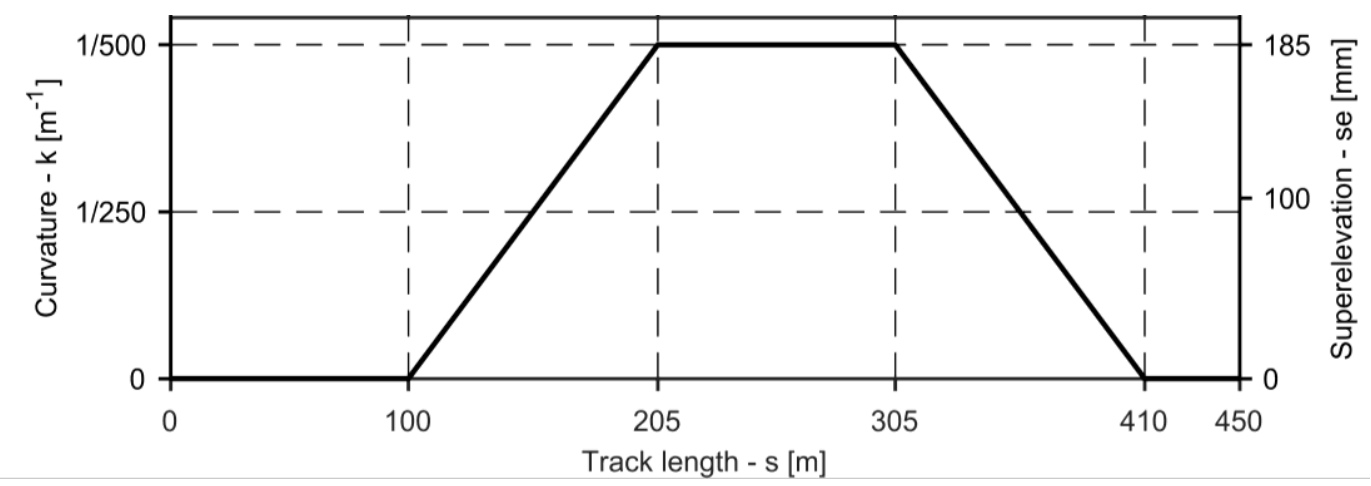

Figure 15: Curvature and superelevation along track length.

The material properties used to build the finite element model of the track are presented on Table 1 , for the rail and sleeper beam elements, and on Table 2, for the remaining spring-damper elements, being the references in which the data for the parameters is obtained provided also.

Table 1: Beam element properties of the track model.

\begin{tabular}{|c|c|c|c|c|}
\hline EB beam element properties & Rail & Ref. & Sleeper & Ref \\
\hline Young Modulus - E [Pa] & $2.10 \times 10^{11}$ & [32] & $3.10 \times 10^{10}$ & [54] \\
\hline Torsion Modulus - G [Pa] & $8.08 \times 10^{10}$ & & $1.50 \times 10^{10}$ & [55] \\
\hline Cross Section Area - $A\left[\mathrm{~m}^{2}\right]$ & $7.67 \times 10^{-3}$ & [56] & $5.6 \times 10^{-2}$ & [54] \\
\hline Polar Moment of Area in $n \zeta$ Plane $-J_{\xi \xi}\left[\mathrm{m}^{4}\right]$ & $3.55 \times 10^{-5}$ & {$[56]$} & $1.71 \times 10^{-3}$ & \\
\hline Second Moment of Area in $\xi \zeta$ Plane $-I_{\eta \eta}\left[\mathrm{m}^{4}\right]$ & $3.04 \times 10^{-5}$ & [56] & $2.60 \times 10^{-4}$ & \\
\hline Second Moment of Area in $\xi \eta$ Plane $-I_{\zeta \zeta}\left[\mathrm{m}^{4}\right]$ & $5.12 \times 10^{-6}$ & {$[56]$} & $1.67 \times 10^{-4}$ & \\
\hline Density $\rho\left[\mathrm{Kg} / \mathrm{m}^{3}\right]$ & 7860 & [35] & 2750 & [32] \\
\hline Rayleigh Damping Parameter $-\alpha\left[\mathrm{s}^{-1}\right]$ & $3.98 \times 10^{-4}$ & & $3.98 \times 10^{-4}$ & \\
\hline Rayleigh Damping Parameter $-\beta[\mathrm{s}]$ & 0.94 & & 0.94 & \\
\hline
\end{tabular}

The track model, which in the case of this demonstration scenario has a length of $500 \mathrm{~m}$, includes energy absorption boundary conditions at the start and end of the track model. The properties of the spring-damper elements used in the start and end of the track, in the longitudinal direction, are presented in Table 2. It should also be noted that although the values for the parameters used to model the track are obtained from State-of-Art references, they do not ensure that the track model dynamic response is that of an existing one. The receptances on the rail above the sleeper and in-between sleepers can be evaluated either to validate the track models against experimental results, if these exist, or to provide typical responses for realistic track models that can be compared with those available in the literature, in particular in the work by Knothe and Grassie [24].

The vehicle model considered in this work is used by a Portuguese railway operator for passenger transport $[60,61]$. The initial position of the bodies of the vehicle model, shown in Figure 1, their masses and inertia properties are listed in Table 3. The primary suspension, responsible for transmitting the forces between the axleboxes and the bogie frame, is shown in Figure 2, being its kinematic and force element parameters described in reference [60,61]. The secondary suspension, responsible for transmitting the forces between the bogie frame and the carbody is also shown in Figure 2, being the data necessary to build its model and the bogie carbody connection found in [60]. The relative motion between the wheelset and axleboxes is 
constrained by tapered rolling bearings. Due to the nature and construction of these bearings, it is assumed here that the revolute joints between the wheelset and axleboxes are representative of their relative kinematics [36].

Table 2: Spring-damper element properties of the track model.

\begin{tabular}{lcccccc}
\hline \multicolumn{1}{c}{ Spring-damper element } & Pads & Ref. & Ballast & Ref. & $\begin{array}{c}\text { Sleeper } \\
\text { Interaction }\end{array}$ & Ref. \\
\hline Vertical Stiffness $-K_{v}[\mathrm{~N} / \mathrm{m}]$ & $1.30 \times 10^{8}$ & {$[57]$} & $6.19 \times 10^{7}$ & {$[58]$} & $5.50 \times 10^{5}$ & {$[59]$} \\
Transversal Stiffness $-K_{t}[\mathrm{~N} / \mathrm{m}]$ & $4.00 \times 10^{7}$ & & $1.00 \times 10^{7}$ & {$[59]$} & $4.05 \times 10^{5}$ & {$[59]$} \\
Longitudinal Stiffness $-K_{l}[\mathrm{~N} / \mathrm{m}]$ & $4.00 \times 10^{7}$ & {$[57]$} & $5.50 \times 10^{5}$ & & $3.92 \times 10^{7}$ & {$[35]$} \\
Longitudinal Rotation Stiffness $-K_{r l}[\mathrm{~N} / \mathrm{m}]$ & $2.00 \times 10^{5}$ & & - & & - & \\
Vertical Damping $-C_{v}[\mathrm{Ns} / \mathrm{m}]$ & $1.50 \times 10^{5}$ & {$[57]$} & $2.94 \times 10^{4}$ & {$[35]$} & $2.94 \times 10^{4}$ & \\
Transversal Damping $-C_{t}[\mathrm{Ns} / \mathrm{m}]$ & $1.00 \times 10^{5}$ & & $2.94 \times 10^{4}$ & & $2.94 \times 10^{4}$ & \\
Longitudinal Damping $-C_{l}[\mathrm{Ns} / \mathrm{m}]$ & $1.00 \times 10^{5}$ & {$[57]$} & $2.94 \times 10^{4}$ & & $2.94 \times 10^{4}$ & {$[35]$} \\
Lumped mass $-m[\mathrm{~kg}]$ & - & & 226.41 & {$[58]$} & - & \\
\hline
\end{tabular}

Table 3: Centre of mass and inertia properties of the bodies considered in the vehicle model.

\begin{tabular}{|c|c|c|c|c|c|}
\hline ID & & Body & $\begin{array}{l}\text { Centre of Mass [m] } \\
(\mathrm{X} / \mathrm{Y} / \mathrm{Z})\end{array}$ & Mass $[\mathrm{kg}]$ & $\begin{array}{c}\text { Moment of Inertia }\left[\mathrm{kg} / \mathrm{m}^{2}\right] \\
(\xi \xi / \eta \eta / \zeta \zeta)\end{array}$ \\
\hline 1 & & Carbody & $11.5000 / 0.000 / 1.432$ & 46200 & $\begin{array}{c}78000 / 2600000 / \\
2600000\end{array}$ \\
\hline 2 & & Bogie frame & $21.000 / 0.000 / 0.448$ & 3000 & $2100 / 2600 / 4800$ \\
\hline 3 & $\frac{0}{2}$ & Front wheelset & $22.350 / 0.000 / 0.445$ & 1800 & $900 / 10 / 900$ \\
\hline 4 & 응 & Front left axlebox & $22.350 / 1.072 / 0.445$ & 10 & $1 / 1 / 1$ \\
\hline 5 & 읻 & Front right axlebox & $22.350 /-1.072 / 0.445$ & 10 & $1 / 1 / 1$ \\
\hline 6 & : & Rear wheelset & $19.650 / 0.000 / 0.445$ & 1800 & $900 / 10 / 900$ \\
\hline 7 & $\underset{0}{\mathbb{0}}$ & Rear left axlebox & $19.650 / 1.072$ / 0.445 & 10 & $1 / 1 / 1$ \\
\hline 8 & - & Rear right axlebox & $19.650 /-1.072 / 0.445$ & 10 & $1 / 1 / 1$ \\
\hline 9 & & Bogie frame & $2.000 / 0.000 / 0.448$ & 3000 & $2100 / 2600 / 4800$ \\
\hline 10 & $\frac{0}{6}$ & Front wheelset & $3.350 / 0.000 / 0.445$ & 1800 & $900 / 10 / 900$ \\
\hline 11 & 요 & Front left axlebox & $3.350 / 1.072 / 0.445$ & 10 & $1 / 1 / 1$ \\
\hline 12 & 이 & Front right axlebox & $3.350 /-1.072 / 0.445$ & 10 & $1 / 1 / 1$ \\
\hline 13 & $\stackrel{气}{=}$ & Rear wheelset & $0.650 / 0.000 / 0.445$ & 1800 & $900 / 10 / 900$ \\
\hline 14 & $\stackrel{\sqrt{2}}{5}$ & Rear left axlebox & $0.650 / 1.072 / 0.445$ & 10 & $1 / 1 / 1$ \\
\hline 15 & & Rear right axlebox & $0.650 /-1.072 / 0.445$ & 10 & $1 / 1 / 1$ \\
\hline
\end{tabular}

\subsection{Results}

The vehicle-track interaction dynamics involves a large set of dynamic responses that is not possible to present concisely in this work. With the purpose of presenting the influence of the flexible track on the vehicle dynamics, the interaction forces due to the wheel-rail contact and the kinematics of the front wheelset of the vehicle are selected as representative responses that allow understanding novel features of the approach proposed. In all that follows, the initial $0.25 \mathrm{~s}$ of any simulation results are discarded, as during this period the dynamics of the system exhibits a transient response while reaching a steady-state operation. The kinematics of the leading wheelset of the vehicle is presented in Figure 16, for the lateral position, in Figure 17, for the attack angle, and in Figure 18, for the vertical position. Comparing the results between the standalone simulation in which the track is considered rigid, denoted by rigid, and the cosimulation with the rigid finite element track model, denoted as co-sim rigid, it is observed a good agreement being their maximum absolute deviation lower than $2.5 \times 10^{-5} \mathrm{~m}$ for the lateral motion, and lower than $8 \times 10^{-8} \mathrm{~m}$ for the vertical motion. Comparing the results between the standalone simulation in which the track is considered rigid, denoted by rigid, and the co- 
simulation with the rigid finite element track model, denoted as co-sim rigid, it is observed a good agreement being their maximum absolute deviation lower than, $2.5 \times 10^{-5} \mathrm{~m}$ for the lateral motion, $4.7 \times 10^{-4}{ }^{\circ}$ for the attack angle, and $8 \times 10^{-8} \mathrm{~m}$ for the vertical motion. Given that the two simulations that consider the rigid track, where one is evaluated in co-simulation, the residual deviation on the results shows that the implemented co-simulation procedure is accurate.

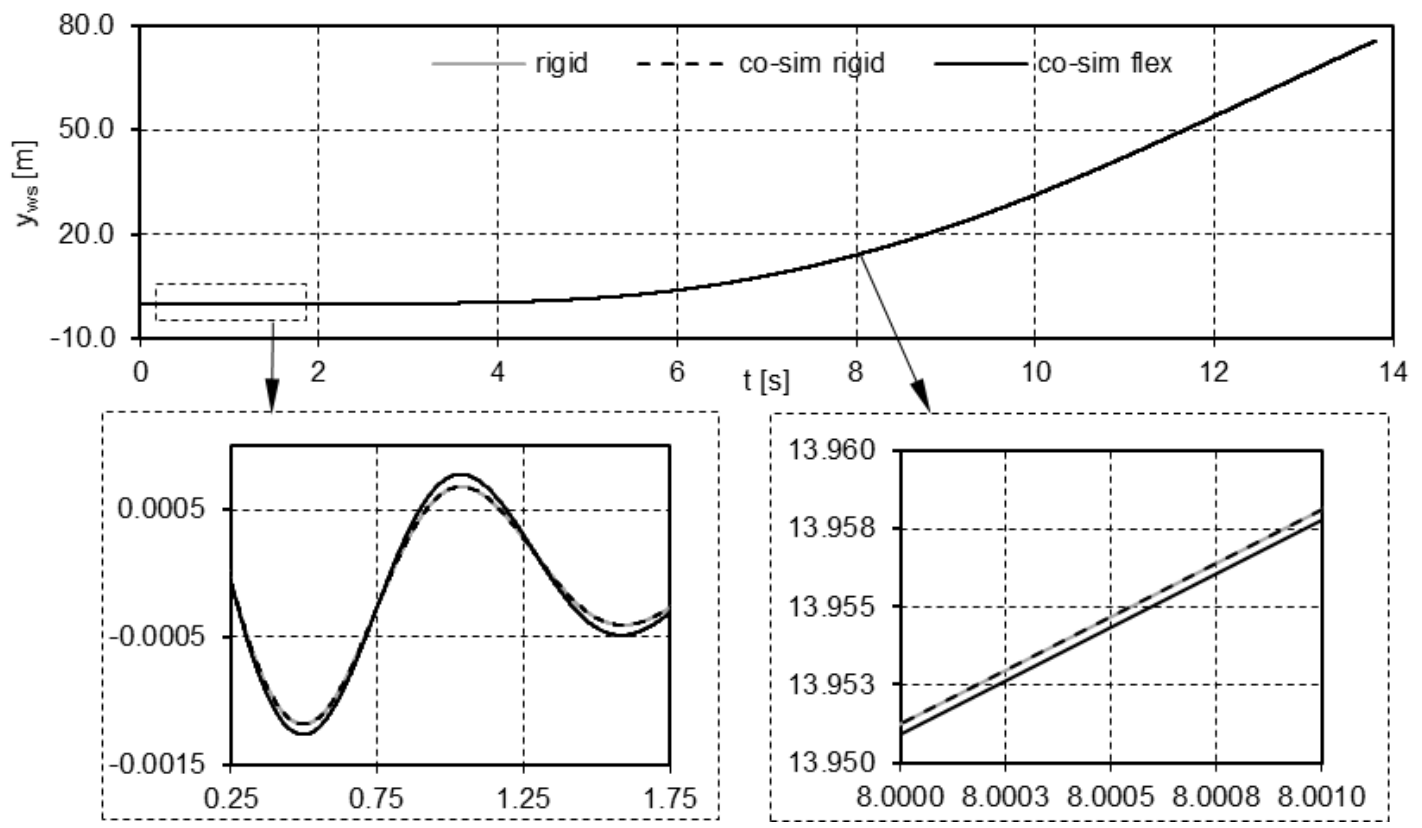

Figure 16: Comparison of the lateral motion of the leading wheelset.

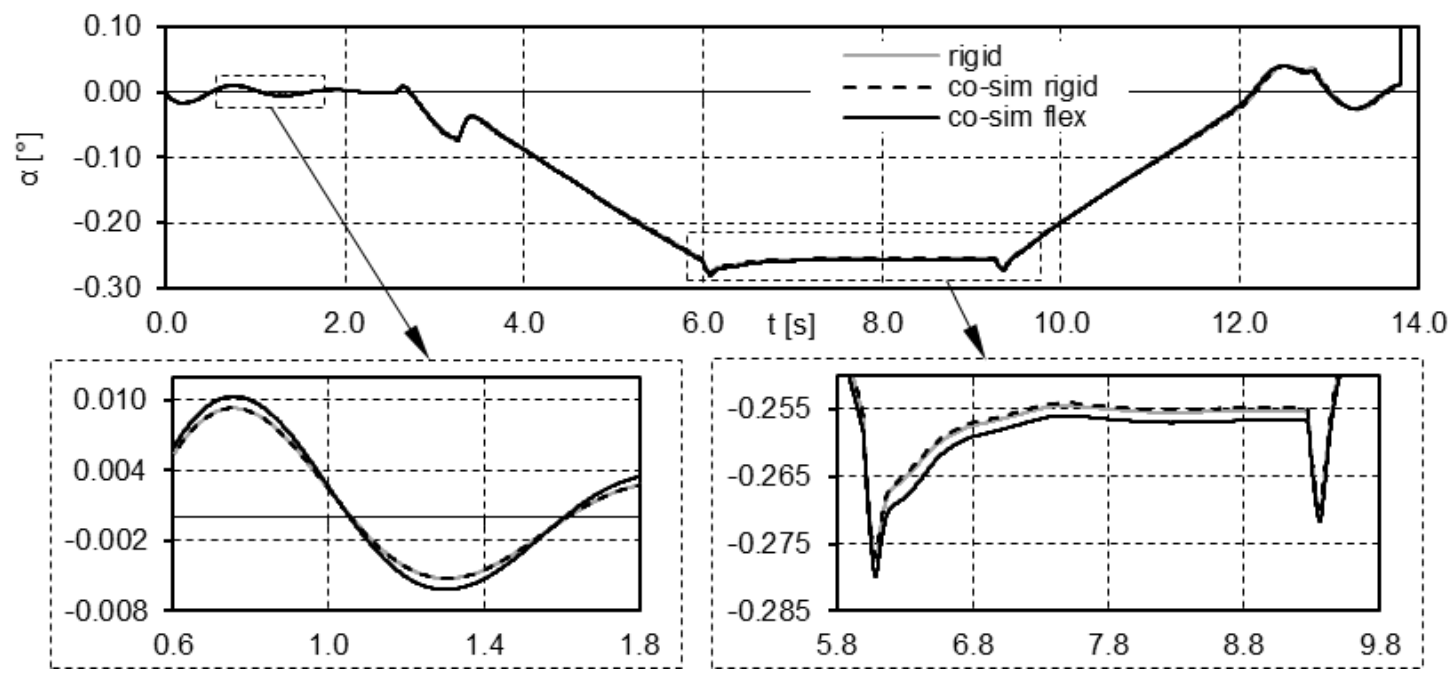

Figure 17: Comparison of the leading wheelset angle of attack.

Comparing the co-simulation results involving the rigid track, co-sim rigid, and the flexible track, co-sim flex, it is possible to identify a distinguishable influence of the track flexibility on the wheelset motion. With respect to the lateral motion, a slightly higher amplitude of the lateral motion is noticeable in the straight segment of the flexible track simulation. In the curved segment the lateral motion of the wheel also presents small offset from the motion when rigid track is considered. The angle of attack of the leading wheelset 
evaluated also shows a small influence of the track flexibility, being slightly larger angle when track flexibility is considered. Moreover, when comparing the vertical motion of the wheelset, in Figure 18, besides the vertical offset also an oscillatory movement is found in the simulation with track flexibility. This additional oscillatory behaviour is more easily identified in the straight segment whereas in the curve the motion of the wheelset set is also influenced by the wheel flange contact at the outer rail. Note that the frequency of these oscillations is about 51 $\mathrm{Hz}$ which is consistent to the periodicity of the track sleepers, spaced at every $0.6 \mathrm{~m}$, for a vehicle traveling at $110 \mathrm{~km} / \mathrm{h}$.

For the standalone simulation with rigid track and the co-simulation with the flexible track the left and right wheel flange contact forces of the leading wheelset are presented in Figure 19. Flange contact only occurs in the outer wheel during curve negotiation. The force peaks observed when the wheels enter the transition and the curve segment are smaller when track flexibility is considered. On the curve section, it can be noted also that the flange force is oscillating at a higher amplitude when the track is considered rigid.

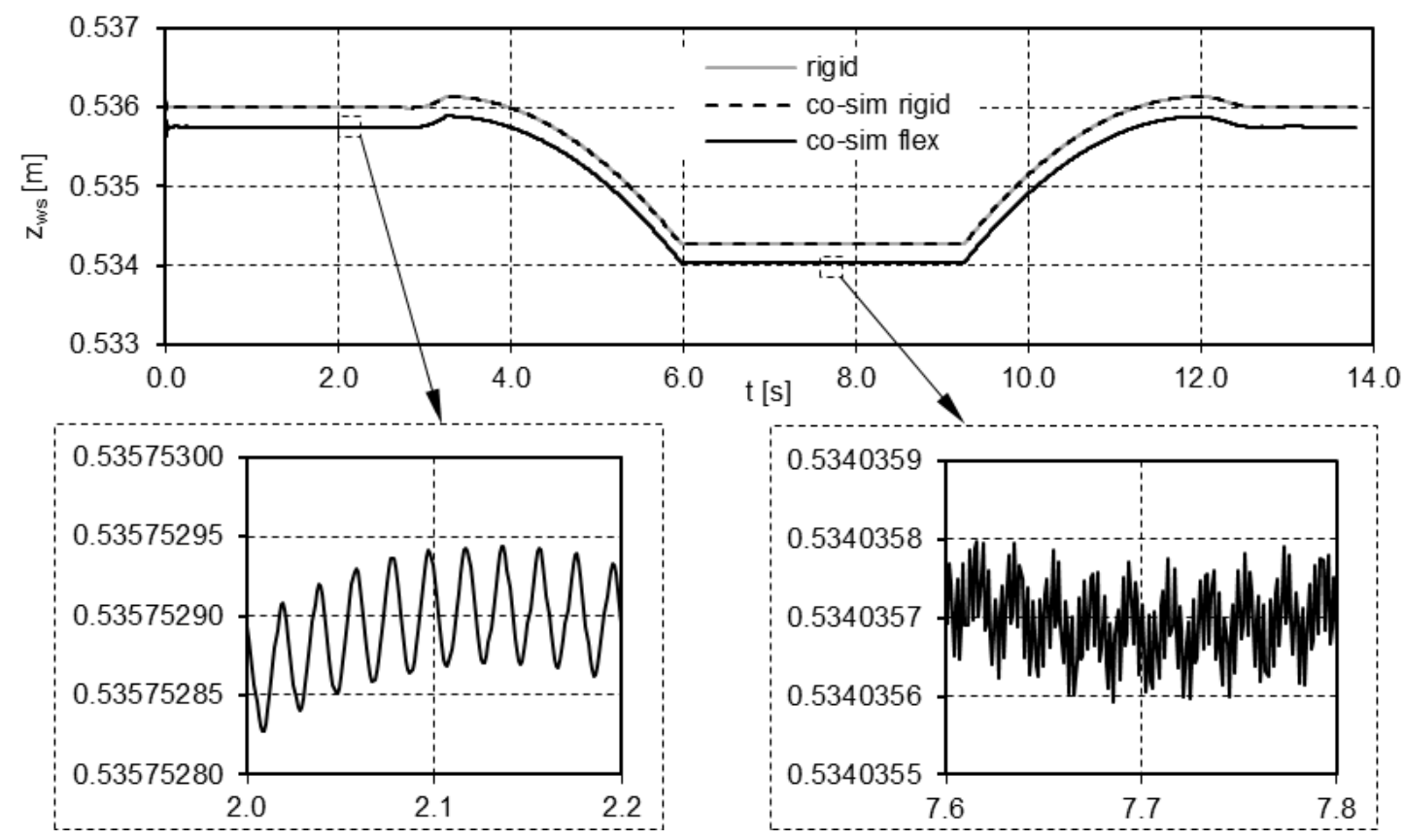

Figure 18: Vertical motion comparison of the leading wheelset.

The oscillating amplitude and peak differences on the curved segment of the track can also be observed on the lateral and vertical contact forces applied on the wheel. These forces are presented in Figure 20 and Figure 21, respectively, for the left and right leading wheels of the front bogie. In the curve segment of the track both the lateral and vertical contact forces of the right wheel are higher. This is due to the flange forces acting on the right wheel when the curve is negotiated. It is also possible to observe two force peaks on the first transition segment from straight to curved track around time $2.5 \mathrm{~s}$ and $3.2 \mathrm{~s}$. These correspond to the instants in which the leading wheel of the front and rear bogies enter the transition zone. Therefore, the wheel-rail contact on the front wheel of the front bogie is sensitive to the contact perceived on the rear bogie. Furthermore, on the straight segment the lateral contact forces from the cosimulation with flexible track are $10 \%$ higher than those observed for the rigid track simulation. This difference can be related with the configuration of the deformed track which promotes different wheel-rail contact conditions. It is also of importance to state that although, for the 
sake of simplicity, the contact forces on the co-simulation with rigid track, co-sim rigid, are not shown here, they are similar to those obtained with the standalone multibody code in which only rigid tracks are used.

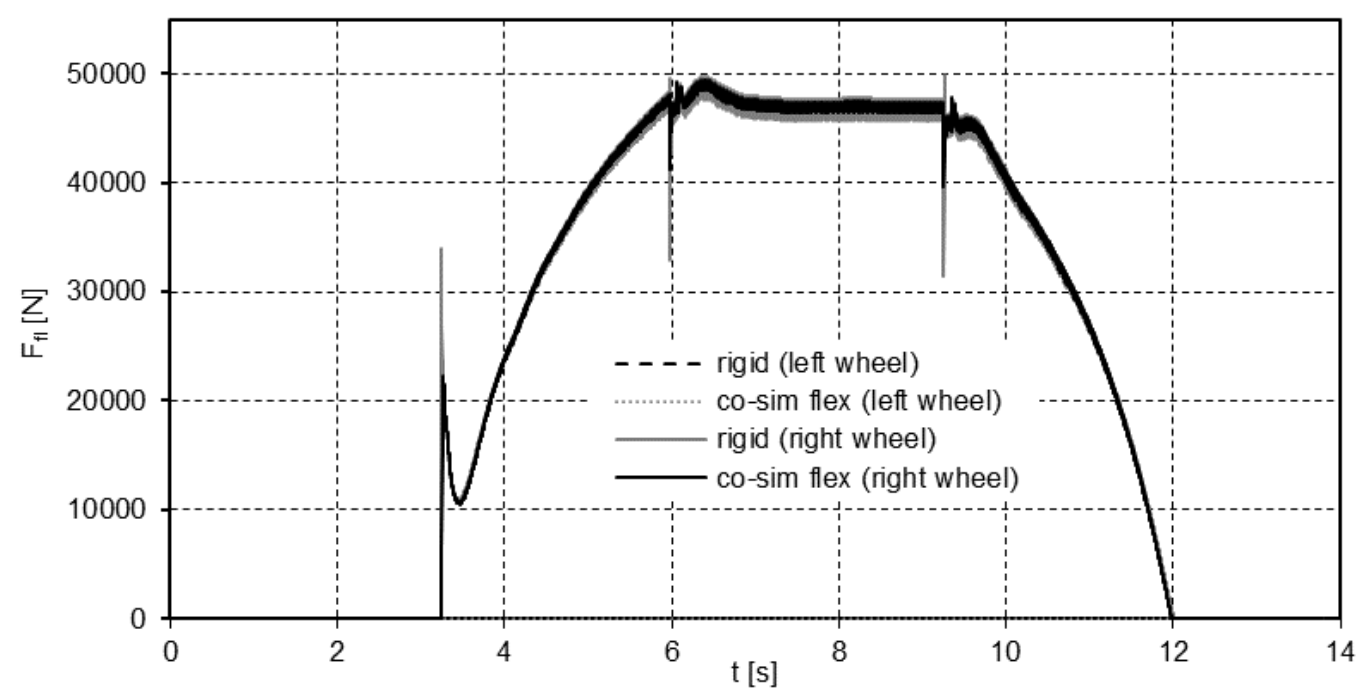

Figure 19: Flange contact force on the left and right wheel of the leading wheelset.

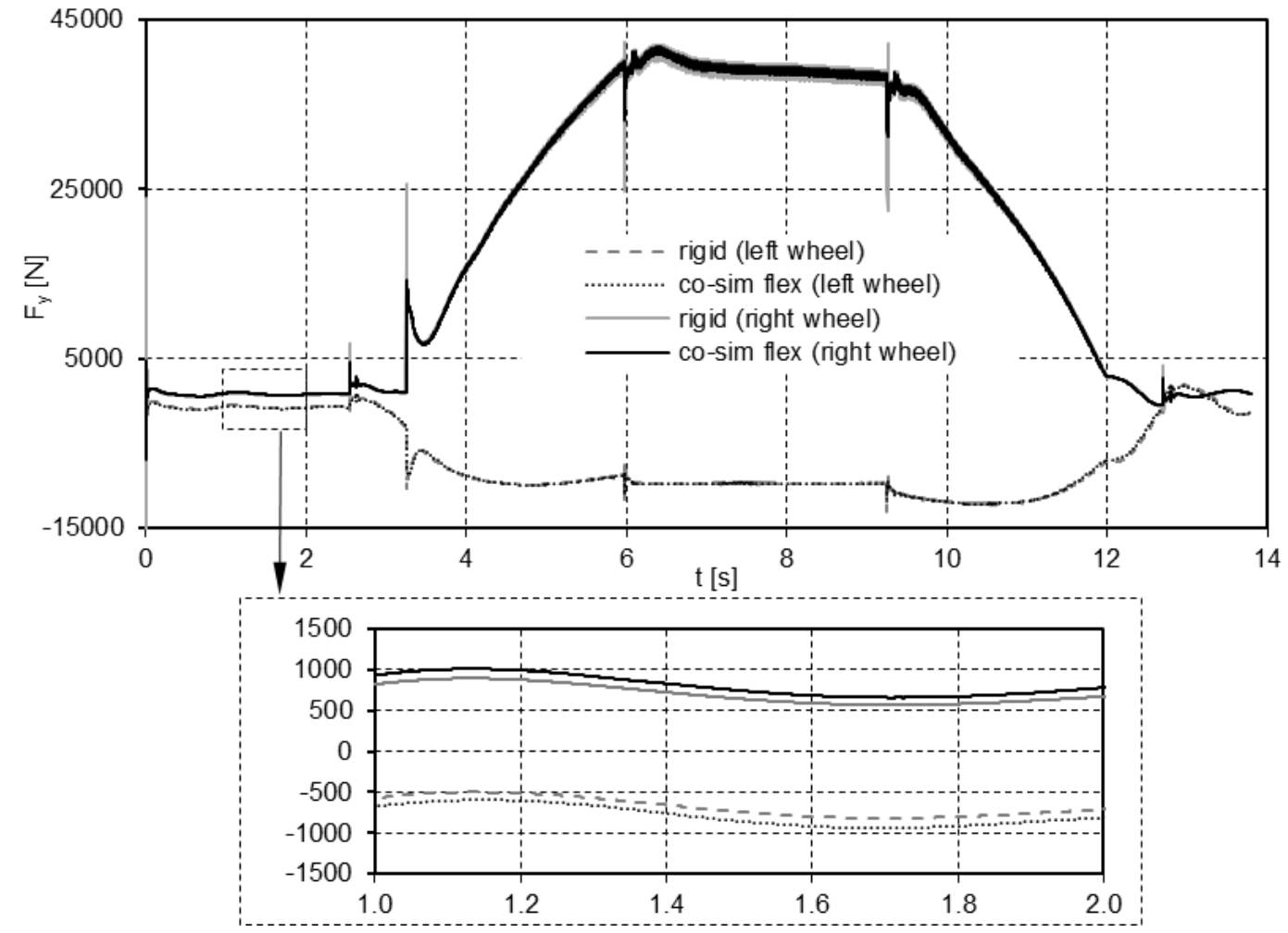

Figure 20: Lateral forces applied on the left and right leading wheel.

The effects of the vehicle-track interaction on the flexible track are depicted by the vertical and transversal displacements of the left and right rail at two different cross-sections, presented in Figure 22 and Figure 23. These figures correspond respectively to the rails displacements evaluated on the straight and curve segment at the 66 and 255 metre mark of the 
track length. The vertical solid lines marked in Figure 22 and Figure 23 indicate the instants in which the train wheels pass on each mark. The absolute maximum displacement peaks are observed in-between the front and rear wheel passage of each bogie, except for the transversal displacements on the curved segment. This relates to the contact on the wheel flange that only occurs on the front right wheel of each bogie. Furthermore, it can be observed that on the same track position each wheel that passes perceives the position of the rail differently, which cannot be represented with a rigid track model. Moreover, the transversal track displacements on the straight segment are symmetric, i.e., the left and right rails move to the inside of the track. Contrarily, on the curved segment both rails are displaced to the outer side of the curve being the right wheel displacement more prominent. This effect is also observed for the vertical displacements in the curved section where the outside rail is loaded heavily due to the curve negotiation and the track superelevation.

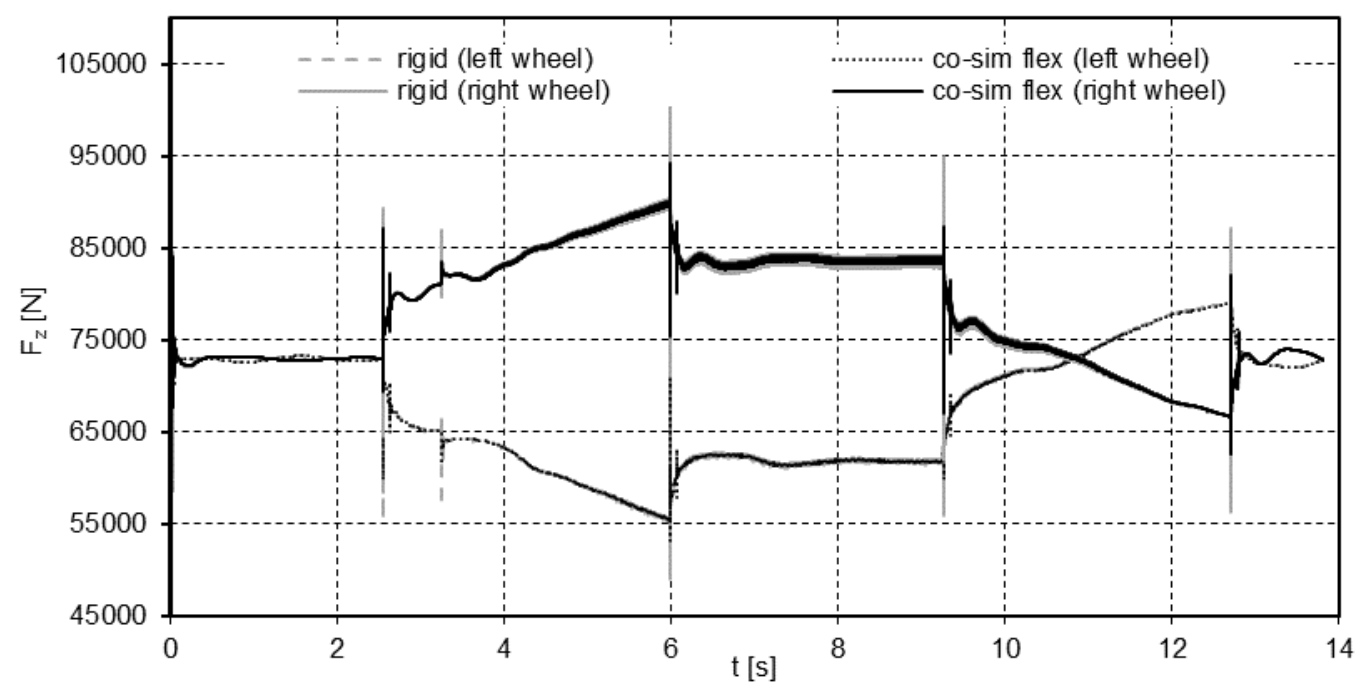

Figure 21: Vertical forces applied on the left and right leading wheel.

In the simulation of the railway vehicle-track interaction scenarios developed in this work the simulation of the dynamics of the vehicle and track multibody model uses a variable time step integrator while a fixed time step of $2 \times 10^{-5} \mathrm{~s}$, used for the finite element flexible track model. This value for the time step is obtained by reducing the step size until the contact forces evaluated stabilize and converge, i.e., until they become identical for any time step smaller than that identified. It should also be noted that the co-simulation with rigid track and flexible track are, respectively, 7.9 and 57.3 times longer than that with the standalone multibody simulation with rigid track. 

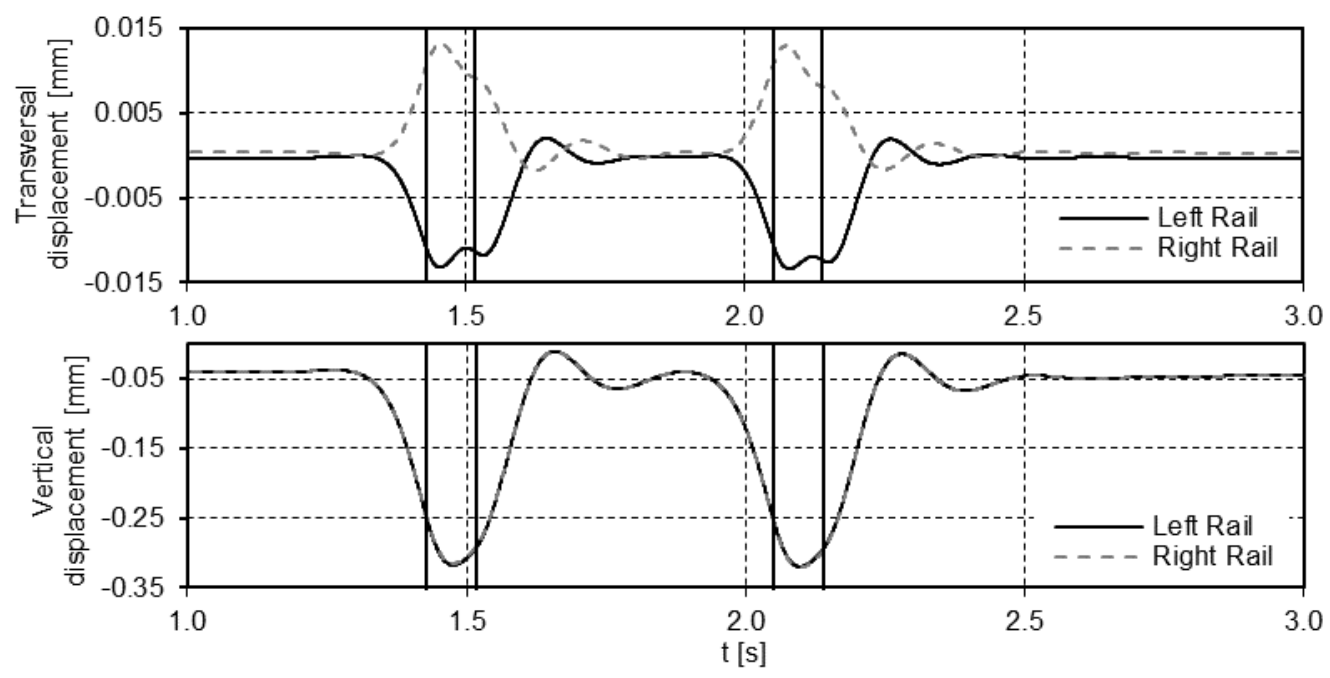

Figure 22: Transversal and vertical displacements of the left and right rail at the 66 metre mark of the track (straight track segment).
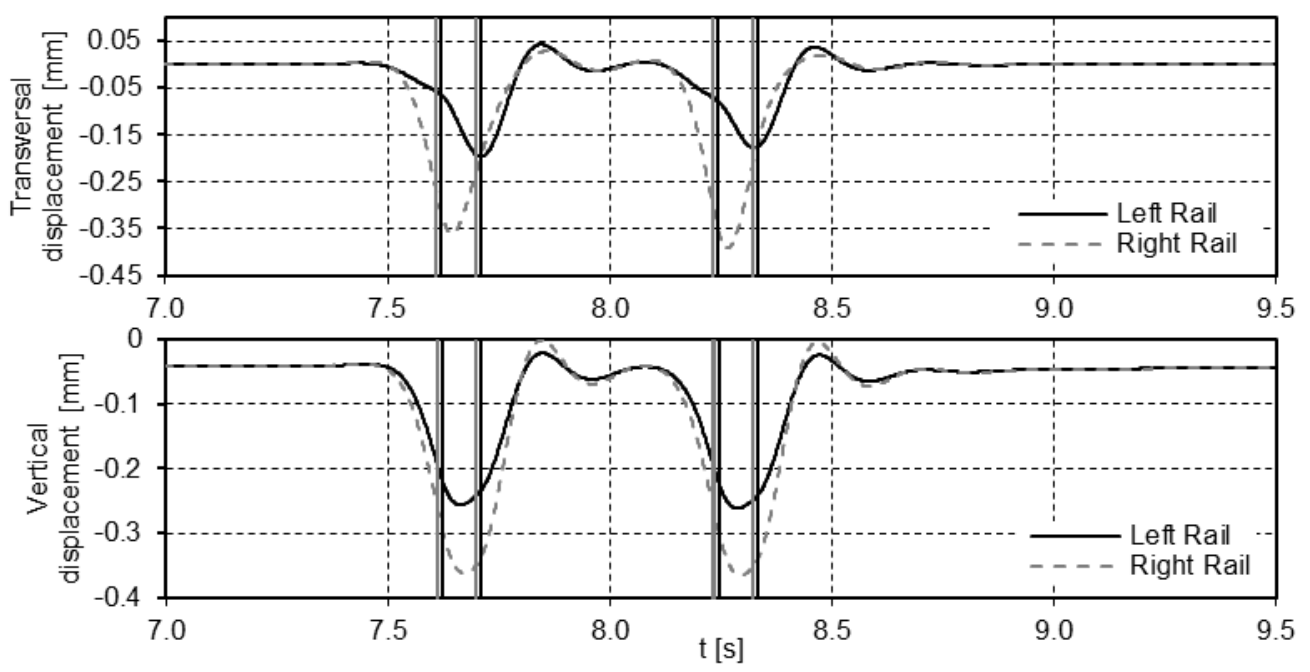

Figure 23: Transversal and vertical displacements of the left and right rail at the 255 metre mark of the track (curve track segment).

\section{Conclusions}

This work proposes a vehicle-track co-simulation methodology to allow the study of the coupled dynamics of the railway vehicle and the flexible track models. The key ingredient of the co-simulation is the wheel-rail interaction characterized by the rolling contact forces in which the contact detection problem is strongly influenced by the ability to evaluate the track deformation. The vehicle model is described and analysed using a multibody dynamics model in which a variable time step integrator is used. The track model is described by a linear finite element method in which a fixed time step integrator, of the Newmark family, is used. The wheel-rail contact force model is evaluated online with the Polach algorithm taking into account the deformation of the rails. The study of a case scenario allows to identify some of the novel features of the methodology proposed here. Not only significant differences on the 
vehicle kinematics exist when considering the track flexibility, namely during curve negotiations, but also the contact forces are modified, being the lateral, or creep, forces higher for a flexible track. The track deformations are clearly identified, and closely related to the train wheelset kinematics, by using the methodology proposed. The results obtained do not allow to understand up to what extend the track flexibility influences the vehicle dynamics. Further studies on this aspect of the vehicle-track coupled dynamics can be carried as the interaction modelling procedure, via co-simulation, shows to be accurate and robust.

\section{REFERENCES}

1. ERRAC: Strategic Rail Research Agenda 2020., Brussels, Belgium (2007)

2. OECD: Strategic Transport Infrastructure Needs to 2030. OECD Publishing, Paris, France (2012)

3. Felippa, C.A., Park, K.C.C., Farhat, C.: Partitioned analysis of coupled mechanical systems. Comput. Methods Appl. Mech. Eng. 190, 3247-3270 (2001). doi:10.1016/S0045-7825(00)00391-1

4. Hulbert, G., Ma, Z.-D., Wang, J.: Gluing for Dynamic Simulation of Distributed Mechanical Systems. In: Ambrósio (Ed.), J. (ed.) Advances on Computational Multibody Systems. pp. 69-94. Springer, Dordrecht, The Netherlands (2005)

5. Kubler, R., Schiehlen, W.: Modular Simulation in Multibody System Dynamics. Multibody Syst. Dyn. 4, 107-127 (2000)

6. Dietz, S., Hippmann, G., Schupp, G.: Interaction of Vehicles and Flexible Tracks by Co-Simulation of Multibody Vehicle Systems and Finite Element Track Models. Veh. Syst. Dyn. 37, 372-384 (2002). doi:10.1080/00423114.2002.11666247

7. Heckmann, A., Arnold, M., Vaculín, O.: A modal multifield approach for an extended flexible body description in multibody dynamics. Multibody Syst. Dyn. 13, 299-322 (2005). doi:10.1007/s11044005-4085-3

8. Liu, F., Cai, J., Zhu, Y., Tsai, H.M., Wong, A.S.F.: Calculation of Wing Flutter by a Coupled FluidStructure Method. J. Aircr. 38, 334-342 (2001). doi:10.2514/2.2766

9. Bathe, K.J., Zhang, H.: Finite element developments for general fluid flows with structural interactions. Int. J. Numer. Methods Eng. 60, 213-232 (2004). doi:10.1002/nme.959

10. Naya, M., Cuadrado, J., Dopico, D., Lugris, U.: An Efficient Unified Method for the Combined Simulation of Multibody and Hydraulic Dynamics: Comparison with Simplified and Co-Integration Approaches. Arch. Mech. Eng. LVIII, 223-243 (2011). doi:10.2478/v10180-011-0016-4

11. Busch, M., Schweizer, B.: Coupled simulation of multibody and finite element systems: an efficient and robust semi-implicit coupling approach. Arch. Appl. Mech. 82, 723-741 (2012). doi:10.1007/s00419011-0586-0

12. Carstens, V., Kemme, R., Schmitt, S.: Coupled simulation of flow-structure interaction in turbomachinery. Aerosp. Sci. Technol. 7, 298-306 (2003). doi:10.1016/S1270-9638(03)00016-6

13. Spreng, F., Eberhard, P., Fleissner, F.: An approach for the coupled simulation of machining processes using multibody system and smoothed particle hydrodynamics algorithms. Theor. Appl. Mech. Lett. 3, 013005 (2013). doi:10.1063/2.1301305

14. Anderson, K.S., Duan, S.: A hybrid parallelizable low-order algorithm for dynamics of multi-rigid-body systems: Part I, chain systems. Math. Comput. Model. 30, 193-215 (1999). doi:10.1016/S08957177(99)00190-9

15. Wang, J., Ma, Z., Hulbert, G.M.: A Gluing Algorithm for Distributed Simulation of Multibody Systems. Nonlinear Dyn. 34, 159-188 (2003). doi:10.1023/B:NODY.0000014558.70434.b0

16. Verhoef, M., Visser, P., Hooman, J., Broenink, J.: Co-simulation of Distributed Embedded Real-Time Control Systems. In: Davies, J. and Gibbons, J. (eds.) Integrated Formal Methods: 6th International Conference, IFM 2007, Oxford, UK, July 2-5, 2007. Proceedings. pp. 639-658. Springer Berlin Heidelberg, Berlin, Heidelberg (2007)

17. Spiryagin, M., Simson, S., Cole, C., Persson, I.: Co-simulation of a mechatronic system using Gensys and Simulink. Veh. Syst. Dyn. 50, 495-507 (2012). doi:10.1080/00423114.2011.598940

18. Gu, B., Asada, H.H.: Co-Simulation of Algebraically Coupled Dynamic Subsystems Without Disclosure of Proprietary Subsystem Models. J. Dyn. Syst. Meas. Control. 126, 1 (2004). doi:10.1115/1.1648307

19. Ambrósio, J., Pombo, J., Rauter, F., Pereira, M.: A Memory Based Communication in the CoSimulation of Multibody and Finite Element Codes for Pantograph-Catenary Interaction Simulation. In: Bottasso C.L., E. (ed.) Multibody Dynamics. pp. 211-231. Springer, Dordrecht, The Netherlands (2008) 
20. Ambrósio, J., Pombo, J., Antunes, P., Pereira, M.: PantoCat statement of method. Veh. Syst. Dyn. 53, 314-328 (2015). doi:10.1080/00423114.2014.969283

21. Massat, J.-P., Laurent, C., Bianchi, J.-P., Balmès, E.: Pantograph catenary dynamic optimisation based on advanced multibody and finite element co-simulation tools. Veh. Syst. Dyn. 52, 338-354 (2014). doi:10.1080/00423114.2014.898780

22. Colombo, E.F., Di Gialleonardo, E., Facchinetti, A., Bruni, S.: Active carbody roll control in railway vehicles using hydraulic actuation. Control Eng. Pract. 31, 24-34 (2014). doi:10.1016/j.conengprac.2014.05.010

23. Kuka, N., Verardi, R., Ariaudo, C., Dolcini, A.: Railway Vehicle Driveline Modelling and CoSimulations in SIMPACK-Simulink. In: Proceedings of the Third International Conference on Railway Technology: Research, Development and Maintenance", Civil-Comp Press, Stirlingshire, UK, 2016. , Cagliari, Sardinia, Italy (2016)

24. Knothe, K.L., Grassie, S.L.: Modelling of Railway Track and Vehicle/Track Interaction at High Frequencies. Veh. Syst. Dyn. 22, 209-262 (1993). doi:10.1080/00423119308969027

25. Pombo, J., Ambrósio, J.: Application of a Wheel-Rail Contact Model to Railway Dynamics in Small Radius Curved Tracks. Multibody Syst. Dyn. 19, 91-114 (2008)

26. Magalhaes, H., Ambrosio, J., Pombo, J.: Railway vehicle modelling for the vehicle-track interaction compatibility analysis. Proc. Inst. Mech. Eng. Part K J. Multi-body Dyn. 230, 251-267 (2016). doi:10.1177/1464419315608275

27. Mazzola, L., Bruni, S.: Effect of suspension parameter uncertainty on the dynamic behaviour of railway vehicles. In: Applied Mechanics and Materials. pp. 177-185. Trans Tech Publ (2012)

28. Polach, O., Evans, J.: Simulations of Running Dynamics for Vehicle Acceptance: Application and Validation. Int. J. Railw. Technol. 2, (2013). doi:10.4203/ijrt.2.4.4

29. Di Gialleonardo, E., Braghin, F., Bruni, S.: The influence of track modelling options on the simulation of rail vehicle dynamics. J. Sound Vib. 331, 4246-4258 (2012). doi:10.1016/J.JSV.2012.04.024

30. Escalona, J.L., Sugiyama, H., Shabana, A. a.: Modelling of structural flexiblity in multibody railroad vehicle systems. Veh. Syst. Dyn. 51, 1027-1058 (2013). doi:10.1080/00423114.2013.786835

31. Lundqvist, A., Dahlberg, T.: Load impact on railway track due to unsupported sleepers. Proc. Inst. Mech. Eng. Part F J. Rail Rapid Transit. 219, 67-77 (2005). doi:10.1243/095440905X8790

32. Recuero, A.M., Escalona, J.L., Shabana, A.A.: Finite-element analysis of unsupported sleepers using three-dimensional wheel-rail contact formulation. Proc. Inst. Mech. Eng. Part K J. Multi-body Dyn. 225, 153-165 (2011). doi:10.1177/2041306810394971

33. Johansson, A., Pålsson, B., Ekh, M., Nielsen, J.C.O., Ander, M.K.A., Brouzoulis, J., Kassa, E.: Simulation of wheel-rail contact and damage in switches and crossings. Wear. 271, 472-481 (2011). doi:10.1016/j.wear.2010.10.014

34. Martínez-Casas, J., Di Gialleonardo, E., Bruni, S., Baeza, L.: A comprehensive model of the railway wheelset-track interaction in curves. J. Sound Vib. 333, 4152-4169 (2014). doi:10.1016/J.JSV.2014.03.032

35. Zhai, W., Wang, K., Cai, C.: Fundamentals of vehicle-track coupled dynamics. Veh. Syst. Dyn. 47, 1349-1376 (2009). doi:10.1080/00423110802621561

36. Nikravesh, P.E.: Computer-Aided Analysis of Mechanical Systems. Prentice-Hall, Englewood Cliffs, New Jersey (1988)

37. Ambrósio, J., Neto, A.: Stabilization Methods for the Integration of DAE in the Presence of Redundant Constraints. Multibody Syst. Dyn. 10, 81-105 (2003)

38. Gear, C.W.: Simultaneous Numerical Solution of Differential-Algebraic Equations. IEEE Trans. Circuit Theory. 18, 89-95 (1971)

39. Przemieniecki, J.S.: Theory of Matrix Structural Analysis. McGraw-Hill, New York (1968)

40. Ambrósio, J., Antunes, P., Pombo, J.J.: On the requirements of interpolating polynomials for path motion constraints. In: Kecskeméthy, A. and Geu Flores, F. (eds.) Mechanisms and Machine Science. pp. 179-197. Springer International Publishing (2015)

41. Pombo, J., Ambrósio, J.: An Alternative Method to Include Track Irregularities in Railway Vehicle Dynamic Analyses. Nonlinear Dyn. 68, 161-176 (2012)

42. Costa, J., Antunes, P., Magalhães, H., Ambrósio, J., Pombo, J.: Development of Flexible Track Models for Railway Vehicle Dynamics Applications. In: Pombo, J. (ed.) Proceedings of the Third International Conference on Railway Technology: Research, Development and Maintenance. Civil-Comp Press, Stirlingshire, UK (2016)

43. Hughes, T.: The Finite Element Method: Linear Static and Dynamic Finite Element Analysis. Prentice- 
Hall, Englewood Cliffs, New Jersey (1987)

44. Bathe, K.-J.: Finite element procedures. Prentice Hall, Englewood Cliffs, N.J. (1996)

45. Newmark, N.: A Method of Computation for Structural Dynamics. ASCE J. Eng. Mech. Div. 85, 67-94 (1959)

46. Pombo, J., Ambrósio, J., Silva, M.: A New Wheel-Rail Contact Model for Railway Dynamics. Veh. Syst. Dyn. 45, 165-189 (2007)

47. Lankarani, H.M., Nikravesh, P.E.: A Contact Force Model with Hysteresis Damping for Impact Analysis of Multibody Systems. AMSE J. Mech. Des. 112, 369-376 (1990)

48. Polach, O.: A Fast Wheel-Rail Forces Calculation Computer Code. Veh. Syst. Dyn. 33, 728-739 (1999)

49. Wen, Z., Wu, L., Li, W., Jin, X., Zhu, M.: Three-dimensional elastic-plastic stress analysis of wheelrail rolling contact. Wear. 271, 426-436 (2011). doi:10.1016/j.wear.2010.10.001

50. Schweizer, B., Li, P., Lu, D., Meyer, T.: Stabilized implicit co-simulation methods: solver coupling based on constitutive laws. (2015)

51. Quinn, M.J.: Parallel Programming in C with MPI and OpenMP. McGraw-Hill Higher Education (2004)

52. Wilkinson, B., Allen, C.M.: Parallel Programming: Techniques and Applications Using Networked Workstations and Parallel Computers. Pearson/Prentice Hall (2005)

53. Downey, A.B.: The Little Book of Semaphores. Science (80-. ). 211, 1-291 (2009). doi:10.1017/CBO9781107415324.004

54. Li, D., Selig, E.T.: Method for Railroad Track Foundation Design. I: Development. J. Geotech. Geoenvironmental Eng. 124, 316-322 (1998). doi:10.1061/(ASCE)1090-0241(1998)124:4(316)

55. SMARTRACK Project - System Dynamics Assessment of Railway Tracks: A Vehicle-Infrastructure Integrated Approach FCT PTDC/EME-PME/101419/2008. (2013)

56. CEN: EN 13674-1 Railway applications - Track - Rail - Part 1: Vignole railway rails 46 kg/m and above, (2011)

57. Zhai, W., Wang, K., Cai, C.: Fundamentals of vehicle-track coupled dynamics. Veh. Syst. Dyn. 47, 1349-1376 (2009). doi:10.1080/00423110802621561

58. Zhai, W.M., Wang, K.Y., Lin, J.H.: Modelling and experiment of railway ballast vibrations. J. Sound Vib. 270, 673-683 (2004). doi:10.1016/S0022-460X(03)00186-X

59. Esveld, C.: Improved knowledge of CWR track. Interact. Conf. cost Eff. Saf. Asp. Railw. track. (1998)

60. Magalhães, H.: Development of Advanced Computational Models of Railway Vehicles - Master Thesis. Instituto Superior Técnico, Lisboa, Portugal (2013)

61. Costa, J.: Railway Dynamics with Flexible Tracks - Master Thesis. Instituto Superior Técnico, Lisboa, Portugal (2015) 